\title{
Reaction-Diffusion Processes, Critical Dynamics and Quantum Chains
}

\author{
Francisco C. Alcaraz ${ }^{a}$, Michel Droz $^{b}$, Malte Henkel $^{b}$ and Vladimir Rittenberg ${ }^{a}$ \\ a Physikalisches Institut, Universität Bonn \\ Nußallee 12, D - 5300 Bonn 1, Germany \\ ${ }^{b}$ Département de Physique Théorique, Université de Genève \\ 24 quai Ernest Ansermet, CH - 1211 Genève 4, Switzerland
}

\section{UGVA-DPT 1992/12-799}

\begin{abstract}
The master equation describing non-equilibrium one-dimensional problems like diffusion limited reactions or critical dynamics of classical spin systems can be written as a Schrödinger equation in which the wave function is the probability distribution and the Hamiltonian is that of a quantum chain with nearest neighbor interactions. Since many one-dimensional quantum chains are integrable, this opens a new field of applications. At the same time physical intuition and probabilistic methods bring new insight into the understanding of the properties of quantum chains. A simple example is the asymmetric diffusion of several species of particles which leads naturally to Hecke algebras and $q$ deformed quantum groups. Many other examples are given. Several relevant technical aspects like critical exponents, correlation functions and finite-size scaling are also discussed in detail.
\end{abstract}

\footnotetext{
${ }^{1}$ Permanent adress: Departamento de Física, Universidade Federal de São Carlos, 13560 São Carlos SP, Brasil
} 


\section{Introduction}

Our understanding of nonequilibrium statistical physics is far behind that for the equilibrium theory. Even simple models may pose a formidable problem if one wants to approach them analytically. In this paper, we shall consider two different types of such problems: the diffusion-limited chemical reactions and the critical dynamics of classical spin systems. It will be shown that in one dimension these problems can be mapped onto quantum chain problems which are often integrable and on which a lot of progress was recently achieved [1]. As a result, many new predictions concerning the nonequilibrium statistical physics problems follow.

The study of the diffusion-limited chemical reactions has stimulated a vast amount of research since the first investigation of Smoluchowski many years ago [2]. Examples

are given by the bimolecular reactions, $A+B \underset{g}{\stackrel{k}{\rightleftharpoons}} C+D$ where the two species $A$ and $B$ diffuse and react to form the two new species $C$ and $D$ and $k$ and $g$ are the forward and backward reaction rates, respectively. The simple case of irreversible reactions for which $g=0, C$ is a inert product and $D$ is not present has been extensively investigated since the original work of Zeldowich [3]. Despite their simplicity those systems exhibit a very rich dynamical behaviour. For homogeneous initial conditions and in low dimensions, the diffusion mechanism is not efficient enough to mix the particles. As a result a spatial segregation occurs and accordingly, a reduced number of reactions between the two species is possible. This results in a slowing down of the dynamical evolution called anomalous kinetics [4]. The evolution of the system is not properly described by the usual rate equations, since the fluctuations play a crucial role. Another interesting situation is when the reactants are initially separated in space; then a reaction-diffusion front is formed during the evolution [5]. Here again the properties of the front are drastically influenced by the fluctuations in low dimensions [6]. We shall restrict ourselves to the homegeneous case here. Several approaches have been used to study such systems: numerical simulations [7], scaling arguments [6] and analytic arguments based on the theory of the Brownian motion [8]. The main results obtained concern the decay of the number of particles of one species 9] and the temporal evolution of the gap developing between the particles of the two species due to the segregation process [10]. However, the analytic results are scarce. Very little is known concerning the behavior of quantities like the space and/or time dependent two-particle correlation functions. Accordingly, new theoretical approches allowing the computation of such quantities are desirable.

Let us first introduce several models which have been studied in the literature:

1. The coagulation model: [11] one considers molecules of one species (say $A$ ), that diffuse in a milieu and react as:

$$
A+A \rightarrow A
$$

2. The annihilation model: [12 the $A$ molecules diffuse and annihilate

$$
A+A \rightarrow \emptyset
$$


where $\emptyset$ denotes an inert state which decouples completely from the dynamics.

3. The two-species trapping reaction: [13] two types of molecules $A$ and $B$ diffuse and one of them, $B$, is "trapped" by $A$ :

$$
A+B \rightarrow A
$$

4. The two-species annihilation reaction: [9] two types of molecules $A$ and $B$ diffuse and annihilate

$$
A+B \rightarrow \emptyset
$$

We are interested in the long-time behaviour of systems like the four examples given above. Considering quantities like the mean concentration of $A$ particles $c_{A}(t)$, in general we expect the following two types of behaviour, as $t \rightarrow \infty$

$$
c_{A}(t) \sim\left\{\begin{array}{c}
t^{-\alpha} \\
\exp -t / \tau
\end{array}\right.
$$

where $\alpha$ is some constant and $\tau$ is known as relaxation time. Throughout this paper, we shall refer to the first type as "massless" or "critical", while the second case will be denoted as "massive". This terminology is borrowed from field theory and equilibrium statistical mechanics. All four reactions defined above have a critical behaviour in the sense that for a given initial concentration of particles, the long time behaviour of the concentrations has an algebraic fall-off. For example, in the reactions (1.1) and (1.2) and in one-dimension, the concentration $c_{A}(t)$ of particles behaves like

$$
c_{A}(t) \sim t^{-1 / 2}
$$

One can allow for reversible (or back) reactions, corresponding to $g \neq 0$. In the long time limit $(t \gg 1 / g)$, a local equilibrium state is reached 114. One is then in a "massive" regime, in which the relaxation towards equilibrium is exponential.

A different type of nonequilibrium problem is the one of critical dynamics of classical spin systems. Let us consider for example the case of a classical Ising model on a lattice. The system is prepared in an initial (nonequilibrium) state. One would like to know how fast the equilibrium state (Gibbs state) will be reached. When not at (static) criticality, the system relaxes exponentially towards equilibrium. In general, the relaxation time $\tau$ scales with the spatial correlation length $\xi$ as the temperature approaches its critical value

$$
\tau \sim \xi^{z}
$$

where $z$ is the dynamical critical exponent. However, counterexamples will be given in this paper where $\xi$ diverges but $\tau$ remains finite (no critical slowing-down!) As these classical spin systems do not have an intrinsic dynamics, the dynamics is thought to come from the interactions between the spins and a heat bath modelling the fast 
degrees of freedom not included in the classical Hamiltonian. The dynamics of these models is thus formulated in terms of a master equation for the probability that a spin configuration is realized at time $t$ [15. Several cases have to be distinguished depending on the presence of macroscopic conserved local quantities. The simplest case is the purely relaxational one in which there are no local conservation laws and we shall restrict to this case throughout the paper. For example, the initial magnetization relaxes towards its equilibrium value. All the physics is put into the transition rates appearing in the master equation. Several choices are possible compatible with the condition of detailed balance, which is the condition insuring that the stationary state will be the Gibbs one. Despite its apparent simplicity, the solution of this problem is very difficult and little can be said analytically even in one dimension [16]. The mapping onto a quantum chain Hamiltonian, which will be explored throughout the paper, will turn out to be a very useful tool to clarify some controversies present in the literature [17], [18].

Both types of nonequilibrium problems (diffusion-limited chemical reactions and critical dynamics) can be described in terms of a master equation for $P(\{\beta\}, t)$, the probability that a configuration $\{\beta\}$ of the systems is realised at time $t$. It turns out that it is suitable to map this master equation problem onto a quantum chain problem [16, 19, 20]. The corresponding equation of motion reads

$$
\partial_{t} P(\{\beta\}, t)=-H P
$$

where $H$ is directly related to the transition rates appearing in the master equation. This will be detailed in Section 3. The question of knowing what kind of dynamical behaviour has the model (power law or exponential relaxation) amounts to know in which phase (massive or massless) of the phase diagram we are.

Moreover, in reaction-diffusion processes $H$ turns out to be non-hermitian and has often the following particular structure

$$
H=H_{0}+H_{1}
$$

where $H_{0}$ is a known integrable Hamiltonian with a larger symmetry than $H$ (for example, in two-states models it can be the XXZ quantum chain in a $Z$ field). $H_{1}$ is non-hermitian and has a lower symmetry but does not affect the spectrum of $H$. Thus, if $H_{0}$ is massless, it follows that $H$ is massless as well. In problems of critical dynamics, $H$ has again often the structure of Eq. (1.9) but with a new meaning. $H$ is now hermitian, $H_{0}$ is again a known Hamiltonian (it can be the same as the one occuring in reaction-diffusion processes) but $H_{1}$ is now a perturbation term, if we approach criticality (small temperatures).

When writing this paper we were faced with two problems. The first one was that we realized that we are left with many more open questions than answers. This is kind of nice because we hope that this is an invitation for other people to look closer at the subject. The second problem concerns the pedagogical presentation of the paper since it addresses two different communities: people doing nonequilibrium 
statistical mechanics and who are familiar with the physical problems treated in this paper but not with integrable systems and those familiar with the Bethe ansatz and two-dimensional field theory but not familiar neither with the physical problems discussed here nor with methods of computing nonequilibrium averages which are different from those techniques used in equilibrium statistical mechanics (vacuum expectation values). We thus suggest two approaches to this paper. One for the "mathematician", the other for the "physicist".

The "mathematician" should start with Appendix C (the last chapter of the paper). There we remind the reader of the definition of the Hecke algebra which depends on a parameter $\beta=q+q^{-1}$ (the significance of $q$ will become apparent immediately). As is well known, if a quantum chain can be written as a sum of generators of a Hecke algebra, through Baxterization [21] one can associate to the chain an integrable vertex model. In this paper, we will consider only chains with $2^{L}$ and $3^{L}$ states $(L$ is the length of the quantum chain). Accordingly, we are going to look for some quotients of the Hecke algebra. To various quotients of the Hecke algebra one can associate a representation given by the $(m / n)$ Perk-Schultz quantum chain [22]. These are chains with $(m+n)^{L}$ states invariant under the quantum superalgebra $U_{q} S U(m / n)$ [23. In this paper, the $(2 / 0),(1 / 1),(3 / 0)$ and $(2 / 1)$ Perk-Schultz models will play a role with $q$ real $(|\beta| \geq 2)$ and the physical significance of the deformation parameter $q$ will become apparent. We also give some new representations of the quotients. First we give non-hermitian representations (for $q$ real), these are relevant for expressions like Eq. (1.9) and next, we give representations of the $(2 / 0)$ and $(1 / 1)$ quotients with $3^{L}$ states. Notice that in the last case the symmetries of the corresponding chain are not anymore $U_{q} S U(2 / 0)$ and $U_{q} S U(1 / 1)$. The knowledge of these symmetries is important because if two chains belong to the same quotient, their spectrum is in general the same but the degeneracies are fixed by the symmetries (a more detailed version of Appendix $\mathrm{C}$ is going to be published elsewhere). After finishing this appendix, the "mathematician" should go through Sections 24 and have a close look at Section 5 where, in the simple case where the calculations can be done using free fermions, one illustrates the peculiar problematics of nonequilibrium statistical mechanics. It is stressed that (see Eq. (1.8)) the knowledge of the wave function (not only the spectrum) plays a crucial role and since in the Bethe ansatz this knowledge is hard to get, the calculation of average quantities presents a new challenge. The "mathematician" should next have a look a Appendices A and B and skip Sections 79 .

We sugggest to the "physicist" to read the paper in the chronological order. In Section 2 we consider quantum chains with $L$ sites (we always take open chains). On each site we take a discrete variable $\beta$ taking $N$ values $(\beta=0$ corresponds to a vacancy). We write the most general master equation describing bimolecular reactions. In Section 3 we write down the corresponding one-dimensional Hamiltonian, see Eq. (1.8). A close related development connecting the master equation in discrete time to the transfer matrix formalism can be found in Refs. [24].

Two-states models are considered in Section 1 . The elementary processes in the master equation describe besides diffusion, annihilation, coagulation and death pro- 
cesses (these processes lead for large times to a state with vacancies only) also the reverse processes creation, decoagulation and birth. We first show that for pure diffusion processes which are left-right asymmetric, the Hamiltonian is just the $q$ deformed XXZ spin-1/2 Heisenberg chain. One discusses in detail the quantum chains corresponding to the different processes and one stresses the importance of the phase diagram of the XXZ Heisenberg chain in a Z magnetic field, especially the Pokrovsky-Talapov line. The learned reader will also notice the importance of nonhermitian representations of the braid group occuring in this type of problems. As is well known when a forward-backward process exists (annihilation and creation for example) through a similarity transformation, the Hamiltonian is hermitian. When all three forward-backward processes are allowed, this is not always possible. We derive the conditions on the rates in oder to get hermitian Hamiltonians.

Section 5 illustrates in detail the simple example of annihilation only, with a rate equal to half the diffusion constant. This corresponds to the physical picture in which, when two particles are on neighboring sites, they always annihilate. As was already known, in this case all the calculations can be done using free fermions. Here we bring some new results. We consider the finite-size scaling of the problem (large time and lattice size with $z=t L^{-2}$ fixed). We show that the finite-size scaling function exists (no logarithmic corrections). This result is important because if one accepts its general validity, it allows numerical estimates of critical exponents like in equilibrium problems. We also compute, for the first time, using the Hamiltonian formalism the density-density correlation function and stress the importance of the scaling limit $r, t \rightarrow \infty$ with $u=r^{2} / t$ fixed.

In Section 6 we consider three-state models (two species of particles and vacancies) with $Z_{3}$ symmetry. Various integrable quantum chains occur which allows us to obtain some rigrous results. Nevertheless, as will be seen much work has still to be done. An interesting three-states model with $Z_{2}$ symmetry is mentioned in Appendix C. In Section 7 we derive and solve the condition on the rates in the master equation which gives chosen steady states. Based on these results, in Sections 8 we discuss the dynamics of the Ising and chiral Potts models. This takes us back to the quantum chains discussed in Sections 1 and 6. We look at the behaviour of the systems when the temperature is small (critical dynamics). This brings us to a problem of perturbation theory. Two cases occur. In the first, although the (spatial) correlation length diverges, the relaxation time stays finite. If we take the corresponding rate to zero and get critical dynamics, then the relaxation time depends on the (spatial) correlation length in a way which is independent of the remaining rates (universality).

In Appendix A we study closer Eq. (1.9). We show how from the knowledge of the eigenvalues and eigenvectors of $H_{0}$ one can compute the eigenvectors of $H$. In Appendix B we consider the example studied in Section 5 from a different point of view. We notice that the non-hermitian Hermiltonian corresponds to a representation of the Hecke algebra and using Baxterization, we derive the corresponding vertex model. It turns out that this is a seven-vertex model. This observation is relevant, since as shown in Appendix C (see also [25]) there are other examples of non-hermitian 
chains (irreversible processes!) which satisfy the Hecke algebra and through this procedure one can find the wave functions using the Bethe ansatz. Section 10 closes the paper with some open questions.

\section{The master equation}

In order to write the master equation which describes a general lattice version of a reaction-diffusion process in one dimension, we take a chain with $L$ sites and at each site $i$ we take a variable $\beta_{i}$ taking $N$ integer values $\left(\beta_{i}=0,1, \ldots, N-1\right)$. By convention we attach the value $\beta_{i}=0$ to a vacancy (inert state). We want to consider a master equation for the probability distribution $P(\{\beta\} ; t)$ with the following form

$$
\begin{aligned}
& \frac{\partial P(\{\beta\} ; t)}{\partial t}=\sum_{k=1}^{L-1}\left[-w_{0,0}^{(k)}\left(\beta_{k}, \beta_{k+1}\right) P\left(\beta_{1}, \ldots, \beta_{L} ; t\right)\right. \\
& \left.\quad+\sum_{\ell, m=0}^{N-1} w_{\ell, m}^{(k)}\left(\beta_{k}, \beta_{k+1}\right) P\left(\beta_{1}, \ldots, \beta_{k}+\ell, \beta_{k+1}+m, \ldots, \beta_{L} ; t\right)\right]
\end{aligned}
$$

where $w_{\ell, m}^{(k)}$ are the transition rates and the prime in the second sum indicates that the pair $\ell=m=0$ should be excluded. We assume all the additions performed on the $\beta_{i}$ to be done modulo $N$.

The advantage of this notation is that one can introduce discrete symmetries in a simple way. We shall assume hereafter that the system is homogenous which implies that the transitions rates are independent of $k$

$$
w_{\ell, m}^{(k)}(\alpha, \beta)=w_{\ell, m}(\alpha, \beta)
$$

for all $k=1, \ldots, L-1$. The probability $\Gamma_{(\alpha, \beta)}^{(\gamma, \delta)}$ that a state $(\gamma, \delta)$ on two consecutive sites will change after an unit time into the state $(\alpha, \beta)$ is

$$
\Gamma_{(\alpha, \beta)}^{(\gamma, \delta)}=w_{\gamma-\alpha, \delta-\beta}(\alpha, \beta) ; \quad(\alpha, \beta) \neq(\gamma, \delta)
$$

The rates $w_{0,0}(\alpha, \beta)$ are related to the probability that in the unit time the state $(\alpha, \beta)$ unchanges. From the conservation of probabilities, we have

$$
w_{0,0}(\alpha, \beta)=\sum_{r, s}^{\prime} w_{r, s}(\alpha-r, \beta-s)
$$

where $r=s=0$ is again excluded.

It is now trivial to check, using Eq. (2.3), that if for the $N$-state model we want to have a $Z_{N}$ symmetry, only the functions

$$
w_{\ell, m}(\alpha, \beta), \quad \ell+m=0(\operatorname{modulo} N)
$$

will appear. This is the case of the annihilation model ( written as $A+A \rightarrow \emptyset+\emptyset$ where $\emptyset$ is a vacancy) which has $Z_{2}$ symmetry $(N=2)$ and for the two-species 
annihilation reaction $(N=3$ and the reaction is written as $A+B \rightarrow \emptyset+\emptyset$ ) which has $Z_{3}$ symmetry. In the former case, we assign to the vacancy the $Z_{2}$ quantum number 0 and to the $A$ molecule the number 1 . In the latter case, the vacancy is denoted by 0 , the $A$ by 1 and the $B$ by 2 .

Parity conservation (left-right symmetry) is achieved if

$$
w_{\ell, m}(\alpha, \beta)=w_{m, \ell}(\beta, \alpha)
$$

Let us comment on the supplementary symmetries besides a possible parity invariance which exists in the four examples given in Section 11. The coagulation model has no symmetry at all. As we have seen, the annihilation model has a $Z_{2}$ symmetry. The two-species trapping reaction has a $U(1)$ symmetry (since the number of $A$ particles is conserved) and a $Z_{2}$ symmetry (give to the vacany and the state $B$ a $Z_{2}$-parity "+" and to the state $A$ a $Z_{2}$-parity "-"). As mentioned before, the two-particle reaction has a $Z_{3}$ symmetry. It has also a supplementary $U(1)$ symmetry, since the difference of the numbers of $A$ and $B$ particles is conserved.

It is often useful to make a change of variables in the master equation. In this paper, we define

$$
P(\{\beta\} ; t)=\Phi(\{\beta\}) \Psi(\{\beta\} ; t)
$$

where $\Phi(\{\beta\})$ takes the special form

$$
\Phi(\{\beta\})=\prod_{k=1}^{L} h^{(k)}\left(\beta_{k}\right)
$$

From Eq. (2.1) $\Psi$ is a solution of the new master equation

$$
\begin{aligned}
\frac{\partial}{\partial t} \Psi(\{\beta\} ; t)= & \sum_{k=1}^{L-1}\left[-w_{0,0}\left(\beta_{k}, \beta_{k+1}\right) \Psi(\{\beta\} ; t)\right. \\
& \left.+\sum_{\ell, m}^{\prime} W_{\ell, m}^{(k)}\left(\beta_{k}, \beta_{k+1}\right) \Psi\left(\beta_{1}, \ldots, \beta_{k}+\ell, \beta_{k+1}+m, \ldots, \beta_{L} ; t\right)\right]
\end{aligned}
$$

with the pair $\ell=m=0$ excluded and

$$
W_{\ell, m}^{(k)}(\alpha, \beta)=\frac{\varphi^{(k)}(\alpha+\ell, \beta+m)}{\varphi^{(k)}(\alpha, \beta)} w_{\ell, m}(\alpha, \beta)
$$

where

$$
\varphi^{(k)}(\alpha, \beta)=h^{(k)}(\alpha) h^{(k+1)}(\beta) ; k=1,2, \ldots, L-1 .
$$

Notice that although the rates $w_{\ell, m}(\alpha, \beta)$ are link independent in general, the $W_{\ell, m}^{(k)}(\alpha, \beta)$ are not. The function $\Phi(\{\beta\})$ has obviously to be non-zero and finite. Let us finally note that if we want all "molecules" to disappear at large times so that we are only left with vacancies, we must have the condition

$$
w_{\ell, m}(-\ell,-m)=0
$$


for $\ell, m=0,1, \ldots, N-1$. One can check that

$$
P(\{\beta\} ; t)=\prod_{k=1}^{L-1} \delta\left(\beta_{k}\right)
$$

satisfies indeed $\partial P / \partial t=0$, with the convention that $\beta_{k}=0$ corresponds to a vacancy.

\section{Quantum chains corresponding to master equa- tions}

We shall now write the master equation (2.9) in the form of a Schrödinger equation. In order to do so, on each site we define a basis in the space of $N \times N$ matrices $E^{k \ell}$. The only non-vanishing matrix element of the matrix $E^{k \ell}$ is the one on the $k^{t h}$ line and the $\ell^{\text {th }}$ column and this matrix element is equal to unity. Assuming homogeneity, the most general $N^{L} \times N^{L}$ Hamiltonian with only nearest-neighbor interactions can be written as

$$
H=\sum_{i=1}^{L-1} H_{i}
$$

where

$$
H_{i}=\sum a_{\ell, m, r, s} E^{\ell m} \otimes E^{r s}
$$

acts in the subspace $V^{(i)} \otimes V^{(i+1)}$ of the $N^{L}$-dimensional vector space. Besides the matrices $E^{k \ell}$, on each site it is also convenient to define the matrix $F$

$$
F=\left(\begin{array}{cccccc}
0 & 1 & 0 & 0 & \cdots & 0 \\
0 & 0 & 1 & 0 & \cdots & 0 \\
\vdots & & & \ddots & & \vdots \\
\vdots & & & & \ddots & \vdots \\
0 & 0 & 0 & \cdots & 0 & 1 \\
1 & 0 & 0 & \cdots & 0 & 0
\end{array}\right) \quad ; \quad F^{N}=1
$$

With these notations, the Hamiltonian density can be written as

$$
H_{i}=U_{i}-T_{i}
$$

where

$$
\begin{aligned}
T_{i} & =\sum_{\ell, m=0}^{N-1} \sum_{\alpha, \beta=0}^{N-1} W_{\ell, m}^{(i)}(\alpha, \beta)\left(E^{\alpha \alpha} F^{\ell}\right) \otimes\left(E^{\beta \beta} F^{m}\right) \\
U_{i} & =\sum_{\alpha, \beta=0}^{N-1} w_{0,0}(\alpha, \beta) E^{\alpha \alpha} \otimes E^{\beta \beta}
\end{aligned}
$$


Here $W_{\ell, m}^{(i)}$ is given in Eq. (2.10) and $w_{0,0}$ in Eq. (2.4). The Schrödinger equation replacing the master equation reads

$$
\frac{\partial}{\partial t}|\Psi\rangle=-H|\Psi\rangle
$$

The Schrödinger equation corresponding to the physical problem (without the similarity transformation Eq. (2.7)) is

$$
\frac{\partial}{\partial t}|P\rangle=-\widetilde{H}|P\rangle
$$

$\widetilde{H}$ is obtained by taking $w_{\ell, m}(\alpha, \beta)$ instead of $W_{\ell, m}(\alpha, \beta)$ in Eq. (3.5). Obviously $H$ and $\widetilde{H}$ have the same spectra but they are given by two different one-dimensional quantum chains which act in a $N^{L}$ Fock space (for $N-1$ species) and their explicit form depends on the chemical reaction and diffusion process. We first discuss the Schrödinger equation (3.8) The ket state $|P\rangle$ is defined as follows 20. Take an orthogonal basis in $\{\beta\}$

$$
|\{\beta\}\rangle=\left|\beta_{1}, \ldots, \beta_{L}\right\rangle ;\left\langle\left\{\beta^{\prime}\right\}||\{\beta\}\right\rangle=\delta_{\left\{\beta^{\prime}\right\},\{\beta\}}
$$

then

$$
|P\rangle=\sum_{\{\beta\}} P(\{\beta\} ; t)|\{\beta\}\rangle
$$

The reaction-diffusion process is determined by the initial $(t=0)$ probability distribution $P_{0}(\{\beta\})$ which defines the "initial" ket state

$$
\left|P_{0}\right\rangle=\sum_{\{\beta\}} P_{0}(\{\beta\})|\{\beta\}\rangle
$$

The Hamiltonian $\widetilde{H}$ is in general non-hermitian and due to probability conservation (Eq. (2.4)), it satisfies the remarkable relation:

$$
\langle 0| \widetilde{H}=0
$$

where the bra ground state $\langle 0|$ is

$$
\langle 0|=\sum_{\{\beta\}}\langle\{\beta\}|
$$

From Eq. (3.12) it follows that the ground state energy is zero. Take now an observable $X(\{\beta\})$ (for example the concentration of $A$ particles in the coagulation model (1.1)). Its average can be computed as follows

$$
\begin{aligned}
<X>(t) & =\sum_{\{\beta\}} X(\{\beta\}) P(\{\beta\} ; t) \\
& =\langle 0|X| P\rangle=\left\langle 0\left|X e^{-\widetilde{H} t}\right| P_{0}\right\rangle
\end{aligned}
$$


Notice that that in nonequilibrium problems one studies the properties of the wave function which is already a probability and not quantum mechanical expectation values

$$
\langle 0|X| 0\rangle
$$

as one does in equilibrium problems. The thermodynamical (continuum) limit can be computed from Eq. (3.14), taking the length $L$ of the chain to infinity for a fixed time $t$. As discussed in detail in Section 5, a second limit (the finite-size scaling limit) is also interesting, where one takes both $t$ and $L$ large but keeps $z=t / L^{2}$ finite.

If $E_{\lambda}$ and $\left|\Psi_{\lambda}\right\rangle$ are the eigenvalues and eigenkets of $\widetilde{H}$, we have from Eq. (3.14)

$$
<X>(t)=\sum_{\lambda} a_{\lambda} e^{-E_{\lambda} t}\left\langle 0|X| \Psi_{\lambda}\right\rangle
$$

where

$$
\left|P_{0}\right\rangle=\sum_{\lambda} a_{\lambda}\left|\Psi_{\lambda}\right\rangle
$$

Thus the large $t$ behaviour of $\langle X\rangle(t)$ is governed by the lowest excitations of $\widetilde{H}$. If, instead of $\widetilde{H}$ we use $H$ (see Eq. (3.7)), then the averages have different expressions

$$
<X>(t)=\sum_{\{\beta\}} X(\{\beta\}) \Phi(\{\beta\}) \Psi(\{\beta\} ; t)=\left\langle 0\left|X \Phi e^{-H t}\right| \Psi_{0}\right\rangle
$$

where

$$
\left|\Psi_{0}\right\rangle=\sum_{\{\beta\}} \Phi^{-1}(\{\beta\}) P_{0}(\{\beta\})|\{\beta\}\rangle
$$

There is another distinctive feature of nonequilibrium problems, as compared to equilibrium ones, and this is the concept of interaction range. As opposed to equilibrium problems where the whole information is only contained in the Hamiltonian (it is nearest-neighbour interactions as can be seen from Eq. (3.1)), in the nonequilibrium case we have to give also $P_{0}(\{\beta\})$. This probability can describe an uncorrelated homogenuous distribution like

$$
P_{0}(\{\beta\})=\prod_{i=1}^{L} f\left(\beta_{i}\right)
$$

or a strongly correlated distribution when, for example, at $t=0$ the reactants are separated in space [6]. The general properties of $P(\{\beta\} ; t)$ and implicitly those of averages (like self-organization, critical dimensions or critical exponents) are going to be different. This can be understood when comparing Eqs. (3.14) and (3.18). Let us assume that we give $\widetilde{H}$ and $P_{0}$ describing a correlated distribution, we can make a similarity transformation to bring $P_{0}$ to an uncorrelated distribution $\Psi_{0}$. After this transformation we will have to work with a new Hamiltonian $H$ (in general with long-range interactions) with different physical properties. 


\section{Two-state Hamiltonians}

Since for two-states models instead of the basis $E^{\alpha, \beta}$ one often prefers the basis of Pauli matrices, let us start by giving some useful identities

$$
\begin{aligned}
E^{01} \otimes E^{10}+E^{10} \otimes E^{01} & =\frac{1}{2}\left(\sigma^{x} \otimes \sigma^{x}+\sigma^{y} \otimes \sigma^{y}\right) \\
E^{01} \otimes E^{01} & =\frac{1}{4}\left(\sigma^{x} \otimes \sigma^{x}-\sigma^{y} \otimes \sigma^{y}+i\left(\sigma^{x} \otimes \sigma^{y}+\sigma^{y} \otimes \sigma^{x}\right)\right) \\
E^{01} \otimes E^{01}+E^{10} \otimes E^{10} & =\frac{1}{2}\left(\sigma^{x} \otimes \sigma^{x}-\sigma^{y} \otimes \sigma^{y}\right)
\end{aligned}
$$

We are now going to consider various Hamiltonians according to their symmetries and chemical properties. In the two-states models, we have $A$ states and vacancies.

\section{1 $Z_{2}$ symmetric, parity non-invariant vacuum-driven pro- cesses}

By a vacuum-driven process, or inert-driven process, we mean reactions which end in a state with vacancies only. This implies from Eq. (2.12)

$$
w_{1,0}(1,0)=w_{0,1}(0,1)=w_{1,1}(1,1)=0
$$

This means that there is no production of pairs of $A$ particles, in other words the process $\emptyset+\emptyset \rightarrow A+A$ is forbidden. Since

$$
w_{1,0}(\alpha, \beta)=w_{0,1}(\alpha, \beta)=0
$$

because of the $Z_{2}$ symmetry, we are left only with the processes

a.) annihilation, with the rate $w_{1,1}(0,0)$

$$
A+A \rightarrow \emptyset+\emptyset
$$

b.) diffusion to the right, with the rate $w_{1,1}(0,1)$

$$
A+\emptyset \rightarrow \emptyset+A
$$

c.) diffusion to the left, with the rate $w_{1,1}(1,0)$

$$
\emptyset+A \rightarrow A+\emptyset
$$

We use now Eqs. (3.4, 3.5, 3.6), take into account that

$$
E^{00} F=E^{01} \quad, \quad E^{11} F=E^{10}, \sigma^{z}=E^{00}-E^{11},
$$


and find

$$
\begin{aligned}
T_{i}= & \frac{\varphi^{(i)}(1,1)}{\varphi^{(i)}(0,0)} w_{1,1}(0,0) E^{01} \otimes E^{01} \\
& +\frac{\varphi^{(i)}(0,1)}{\varphi^{(i)}(1,0)} w_{1,1}(1,0) E^{10} \otimes E^{01}+\frac{\varphi^{(i)}(1,0)}{\varphi^{(i)}(0,1)} w_{1,1}(0,1) E^{01} \otimes E^{10} \\
U_{i}= & \frac{1}{4}\left(w_{1,1}(0,1)+w_{1,1}(1,0)+w_{1,1}(0,0)\right) \mathbf{1} \otimes \mathbf{1} \\
& +\frac{1}{4}\left(w_{1,1}(0,0)-w_{1,1}(0,1)-w_{1,1}(1,0)\right) \sigma^{z} \otimes \sigma^{z} \\
& -\frac{1}{4} w_{1,1}(0,0)\left(\sigma^{z} \otimes \mathbf{1}+\mathbf{1} \otimes \sigma^{z}\right) \\
& +\frac{1}{4}\left(w_{1,1}(1,0)-w_{1,1}(0,1)\right)\left(\sigma^{z} \otimes \mathbf{1}-\mathbf{1} \otimes \sigma^{z}\right)
\end{aligned}
$$

Up to this point we still have freedom in the choice of the function $\varphi^{(k)}\left(\beta_{k}, \beta_{k+1}\right)$ in Eq. (2.8). We now take

$$
\frac{\varphi^{(k)}(1,0)}{\varphi^{(k)}(0,1)}=\sqrt{\frac{w_{1,1}(1,0)}{w_{1,1}(0,1)}}=q ; k=1,2, \ldots, L-1,
$$

and it is clear that $q$ is real since the rates are real. From Eqs. (4.9) and (2.11) we get

$$
\frac{h^{(k)}(1)}{h^{(k)}(0)}=q^{1-k} \lambda^{-1}
$$

where $\lambda$ is an arbitrary parameter. The diffusion constant $D$, expressed as

$$
D=\sqrt{w_{1,1}(0,1) w_{1,1}(1,0)}
$$

fixes the time scale. We then finally obtain

$$
H=D\left(H_{0}+H_{1}\right)
$$

where

$$
\begin{aligned}
& H_{0}=-\frac{1}{2} \sum_{i=1}^{L-1}\left[\sigma_{i}^{x} \sigma_{i+1}^{x}+\sigma_{i}^{y} \sigma_{i+1}^{y}+\Delta \sigma_{i}^{z} \sigma_{i+1}^{z}+\left(1-\Delta^{\prime}\right)\left(\sigma_{i}^{z}+\sigma_{i+1}^{z}\right)\right. \\
&\left.-\frac{1}{2}\left(q-q^{-1}\right)\left(\sigma_{i}^{z}-\sigma_{i+1}^{z}\right)+2 \Delta^{\prime}-\Delta-2\right] \\
& H_{1}=-\frac{w_{1,1}(0,0)}{\lambda^{2} D} \sum_{i=1}^{L-1} q^{1-2 i} E_{i}^{01} E_{i+1}^{01} \\
& \Delta=\frac{q+q^{-1}}{2}-\frac{w_{1,1}(0,0)}{2 D}, \Delta^{\prime}=1-\frac{w_{1,1}(0,0)}{2 D}
\end{aligned}
$$


Let us now discuss the structure of this Hamiltonian. $H_{0}$ is hermitian, $U(1)$ symmetric and its properties are going to be discussed shortly. $H_{1}$ is non-hermitian and has only a $Z_{2}$ symmetry, corresponding to the transformations

$$
\sigma_{i}^{x} \rightarrow-\sigma_{i}^{x} \quad, \quad \sigma_{i}^{y} \rightarrow-\sigma_{i}^{y} \quad, \quad \sigma_{i}^{z} \rightarrow \sigma_{i}^{z}
$$

which makes $H$ only $Z_{2}$-symmetric (as expected) and non-hermitian.

Let us discuss the case of pure diffusion, that is $w_{1,1}(0,0)=0$. We have $H_{1}=0$ and

$$
H_{0}=-\frac{1}{2} \sum_{i=1}^{L-1}\left[\sigma_{i}^{x} \sigma_{i+1}^{x}+\sigma_{i}^{y} \sigma_{i+1}^{y}+\frac{q+q^{-1}}{2} \sigma_{i}^{z} \sigma_{i+1}^{z}-\frac{q-q^{-1}}{2}\left(\sigma_{i}^{z}-\sigma_{i+1}^{z}\right)-\frac{q+q^{-1}}{2}\right]
$$

This Hamiltonian is the $U_{q} S U(2)$ symmetric Hamiltonian of Pasquier and Saleur [26] where the deformation parameter $q$ has a clear physical interpretation, see Eq. (4.9). Moreover, as shown in Appendix $\mathrm{C}, H_{0}$ is the sum of generators of a given quotient of a Hecke algebra (called a Temperley-Lieb algebra). As discussed in detail in Appendix $\mathrm{C}$, for any number of species, asymmetric diffusion defines various quotients of Hecke algebras (we think this is an important observation). As a corollary one can show that for any number of species asymmetric diffusion is always massive.

We now consider the case $w_{1,1}(0,0) \neq 0$, that is the full nonhermitian Hamiltonian Eq. (4.13) which has a special property. From the expression (4.15) of $H_{1}$ we see that one can take the factor $\lambda^{2}$ arbitrary without changing the spectrum. This is certainly so, since this factor only serves to parametrize a similarity transformation. On the other hand, one can also see by direct inspection of the matrix elements of $H$ that the characteristic polynomial of $H$ is not affected by the existence of $H_{1}$. Let us briefly present the argument since we are going to use this repeatedly later on. If $C$ and $D$ are square matrices and if $X$ is a rectangular matrix, it is well known that

$$
\operatorname{det}\left(\begin{array}{cc}
C & X \\
0 & D
\end{array}\right)=\operatorname{det} C \operatorname{det} D
$$

Now, $H_{0}$ has a $U(1)$ symmetry and has thus a block-diagonal form. The corresponding quantum number labelling the blocks is the number of $A$ particles present. Acting with $H_{1}$ reduces this quantum number by two and this plays the role of $X$ in Eq. (4.18). Thus the above determinant formula can be applied and the independence of the spectrum of $H$ from the operator $H_{1}$ follows.

Let us choose $w_{1,1}(0,0)$ such that $\Delta=0$. This corresponds accidentally to the choice of all the previous numerical simulation studies of the one-species annihilation process where one took $q=1$ and $w_{1,1}(0,0)=2 D$. In this case $H$ can be studied in terms of free fermions. This will be done in Section 5 where some new results are presented. In particular we find that for $q=1, H_{0}$ can be written in terms of fermionic number operators as follows

$$
H_{0} \sim \sum_{k}\left(\frac{k}{L}\right)^{2} a_{k}^{+} a_{k}
$$


which describes a Pokrovsky-Talapov phase transition [27]. Notice the quadratic dispersion relation which is expected in any diffusion problem.

In Appendix B we consider a complementary point of view which is related to the problem of integrability of quantum chains with the structure Eq. (4.12) in which $H_{0}$ is integrable (in the present case it corresponds to a six-vertex model) and $H_{1}$ does not affect the spectrum of $H$. We show that $H$ is related to a seven-vertex model for which the Yang-Baxter equations are valid and hence $H$ is integrable.

We now consider the case $\Delta \neq 0$. This brings us, see Eq. (4.13), to discuss properties of the Hamiltonian

$$
H^{\prime}=-\frac{1}{2} \sum_{i=1}^{L-1}\left[\sigma_{i}^{x} \sigma_{i+1}^{x}+\sigma_{i}^{y} \sigma_{i+1}^{y}+\Delta \sigma_{i}^{z} \sigma_{i+1}^{z}+h\left(\sigma_{i}^{z}+\sigma_{i+1}^{z}\right)\right]
$$

This Hamiltonian is integrable [28] and its phase diagram is shown in Fig. 1. For $h=0$, the system is massive with a ferromagnetic ground state if $\Delta>1$, massless and conformally invariant if $-1 \leq \Delta \leq 1$ and again massive with an antiferromagnetic ground state if $\Delta<-1$. For a given $h$, the system is massive ferromagnetic if $\Delta>1-h$, then undergoes a Pokrovsky-Talapov transition at $\Delta_{P T}=1-h$, is in a massless incommensurate phase for $\Delta_{c}<\Delta<1-h$ and reaches again the massive antiferromagnetic phase if $\Delta<\Delta_{c}$.

It can be shown [29] that in the continuum, along the Pokrovsky-Talapov line, the spectrum of $H^{\prime}$ is, up to normalisation, given by Eq. (4.19) for any $\Delta$. In other words, the system is massless with a quadratic dispersion in the momentum $k$.

With this knowledge in hand, let us discuss some properties of $H_{0}$. Since $\Delta^{\prime}<\Delta$, we have $h>1-\Delta$ and the system is massive. If however, $q=1$, we have $\Delta^{\prime}=\Delta$ and we are on the Pokrovsky-Talapov line where the system is massless. There are some immediate questions to ask about this system. If we define

$$
\epsilon=\frac{q+q^{-1}}{2}-1
$$

and if $\tau$ denotes the relaxation time, we are interested in the exponent $\eta$

$$
\tau \sim \epsilon^{-\eta}
$$

It can be shown that $\eta=1$ is independent of $\Delta$ using the standard lore of Hecke algebras, see Appendix C. This result is to be expected since we can change the value of $\Delta$ by changing $D$, see Eq. (4.15). However, changing $D$ merely changes the time scale. More generally, the full finite-size scaling form should read

$$
\tau=L^{\eta_{1}} F(x), \quad x=\epsilon L^{\eta_{2}}
$$

in the simultaneous limit $\epsilon \rightarrow 0, L \rightarrow \infty$ with $x$ kept fixed. Concerning the concentration of $A$ particles

$$
c_{A}=\frac{1}{L} \sum_{i=1}^{L} E_{i}^{11}
$$


in the critical regime $(q=1)$, we are interested in

$$
<c_{A}>(t)=L^{x} \phi(z), z=t L^{-2}
$$

for both $t$ and $L$ being large with $z$ fixed. The exponent 2 in the definition of $z$ was taken because of the quadratic dispersion relation Eq. (4.19). In Section 5 we shall find $x=-1$. Similarly, correlation functions can be introduced and calculated. We shall present the analysis in Section 5 for the case $\Delta=0$. For the case $\Delta \neq 0$, although we expect on physical grounds the same results, the explicit proof is still missing. The Bethe ansatz equations for the wave equations are known only for $H_{0}$ but not for $H$. In Appendix $\mathrm{A}$ it is shown how in principle the knowledge of the eigenfunctions of $H_{0}$ can help to find the eigenfunctions of $H$. In a different approach one could start with the seven-vertex model (which is not limited to the case $\Delta=0$ ), and perform the Bethe ansatz there. The wave functions thus found could be used for $H$. Whether this whole program is manageable remains to be seen.

\section{2 $Z_{2}$ non-invariant, parity invariant vacuum-driven pro- cesses}

From now on, we take always the left-right symmetric case, see Eq. (2.6), in all reaction rates, in particular $w_{1,1}(0,1)=w_{1,1}(1,0)=D$. We choose units of time such that $D=1$. To the processes studied before we now add the following

d.) coagulation, with rate $w_{1,0}(0,1)$

$$
A+A \rightarrow \emptyset+A
$$

e.) decoagulation, with rate $w_{1,0}(1,1)$

$$
\emptyset+A \rightarrow A+A
$$

f.) death, with rate $w_{1,0}(0,0)$

$$
A+\emptyset \rightarrow \emptyset+\emptyset
$$

We take $\varphi^{(i)}(0,1)=\varphi^{(i)}(1,0)(i=1,2, \ldots, L-1)$ and get

$$
\begin{aligned}
T_{i}= & \left(E^{01} \otimes E^{10}+E^{10} \otimes E^{01}\right)+\frac{\varphi^{(i)}(1,1)}{\varphi^{(i)}(0,0)} w_{1,1}(0,0) E^{01} \otimes E^{01} \\
& +\frac{\varphi^{(i)}(1,0)}{\varphi^{(i)}(0,0)} w_{1,0}(0,0)\left(E^{01} \otimes E^{00}+E^{00} \otimes E^{01}\right) \\
& +\frac{\varphi^{(i)}(1,1)}{\varphi^{(i)}(1,0)} w_{1,0}(0,1)\left(E^{01} \otimes E^{11}+E^{11} \otimes E^{01}\right) \\
& +\frac{\varphi^{(i)}(1,0)}{\varphi^{(i)}(1,1)} w_{1,0}(1,1)\left(E^{10} \otimes E^{11}+E^{11} \otimes E^{10}\right)
\end{aligned}
$$




$$
\begin{aligned}
U_{i}= & \frac{1}{2} w_{0,0}(1,0)+\frac{1}{4} w_{0,0}(1,1)+\left(\frac{w_{0,0}(1,1)}{4}-\frac{w_{0,0}(1,0)}{2}\right) \sigma^{z} \otimes \sigma^{z} \\
& -\frac{w_{0,0}(1,1)}{4}\left(\mathbf{1} \otimes \sigma^{z}+\sigma^{z} \otimes \mathbf{1}\right)
\end{aligned}
$$

where

$$
\begin{aligned}
& w_{0,0}(1,1)=2 w_{1,0}(0,1)+w_{1,1}(0,0) \\
& w_{0,0}(1,0)=w_{1,0}(0,0)+w_{1,0}(1,1)+1
\end{aligned}
$$

Thus the Hamiltonian depends on four parameters. Two cases have to be considered separately. In the first case we have only coagulation, in the second case we have coagulation and decoagulation. If we have no decoagulation, $w_{1,0}(1,1)=0$. Then

$$
H=H_{0}+H_{1}
$$

where

$$
\begin{gathered}
H_{0}=-\frac{1}{2} \sum_{i=1}^{L-1}\left[\sigma_{i}^{x} \sigma_{i+1}^{x}+\sigma_{i}^{y} \sigma_{i+1}^{y}+\Delta \sigma_{i}^{z} \sigma_{i+1}^{z}+\left(1-\Delta^{\prime}\right)\left(\sigma_{i}^{z}+\sigma_{i+1}^{z}\right)+2 \Delta^{\prime}-\Delta-2\right] \\
H_{1}=-\sum_{i=1}^{L-1}\left[\frac{\varphi^{(i)}(1,1)}{\varphi^{(i)}(0,0)} w_{1,1}(0,0) E_{i}^{01} E_{i+1}^{01}+\frac{\varphi^{(i)}(1,0)}{\varphi^{(i)}(0,0)} w_{1,0}(0,0)\left(E_{i}^{01} E_{i+1}^{00}+E_{i}^{00} E_{i+1}^{01}\right)\right. \\
\left.+\frac{\varphi^{(i)}(1,1)}{\varphi^{(i)}(1,0)} w_{1,0}(0,1)\left(E_{i}^{01} E_{i+1}^{11}+E_{i}^{11} E_{i+1}^{01}\right)\right] \\
\Delta^{\prime}=1-w_{1,0}(0,1)-\frac{1}{2} w_{1,1}(0,0) \\
\Delta=\Delta^{\prime}+w_{1,0}(0,0)
\end{gathered}
$$

We have checked using the same arguments as above that the spectrum of $H$ is independent of the presence of $H_{1}$. Since $\Delta^{\prime}<\Delta$, we are in a massive phase. The system is massless, i.e. $\Delta=\Delta^{\prime}$, if $w_{1,0}(0,0)=0$, corresponding to the absence of death processes. A straightforward calculation shows that the relaxation time $\tau$ scales like

$$
\tau \sim\left(\frac{w_{1,0}(0,1)}{w_{1,1}(1,0)}\right)^{-1}
$$

This can be seen by repeating the same arguments as for Eq. (4.22). The result Eq. (4.37) can be understood easily if one keeps in mind the time evolution of the system. If one considers the later stages of the process when few "molecules" are left, few of them will meet and annihilate. On the other hand, any one of them can "die" since this is an individual process. 
In the absence of the death process some results are already known for the pure annihilation process [12 or the coagulation process [11] and it remains to be seen how much more can be learnt using the quantum chain formulation.

We now consider the case when the coagulation and the decoagulation processes coexist and we have all terms in Eq. (4.29). Then little can be said. One interesting case [30] is when apart from just diffusion, only coagulation and decoagulation are present, that is $w_{1,1}(0,0)=w_{1,0}(0,0)=0$. We choose

$$
\frac{\varphi^{(i)}(1,1)}{\varphi^{(i)}(1,0)}=\sqrt{\frac{w_{1,0}(1,1)}{w_{1,0}(0,1)}}, \quad(i=1,2, \ldots, L-1) .
$$

We stress that this transformation is singular when the decoagulation rate $w_{1,0}(1,1)$ goes to zero. We get a hermitian Hamiltonian $H=H_{0}+H_{1}$ with $H_{0}$ being given by Eq. (4.33) with

$$
\begin{aligned}
\Delta^{\prime} & =1-w_{1,0}(0,1) \\
\Delta & =\Delta^{\prime}+w_{1,0}(1,1)
\end{aligned}
$$

and

$$
H_{1}=-\sum_{i=1}^{L-1} \sqrt{\left(\Delta-\Delta^{\prime}\right)\left(1-\Delta^{\prime}\right)}\left(\sigma_{i}^{x} E_{i+1}^{11}+E_{i}^{11} \sigma_{i+1}^{x}\right)
$$

where $\Delta^{\prime}<\Delta$ and again we suspect a massive phase, at least when $\Delta^{\prime}$ is close to $\Delta$. Note that now $H_{1}$ is hermitian and its presence does change the spectrum of $H$. This change is in fact quite important. If we look directly at the master equation, besides the trivial stationary probability distribution Eq. (2.13) there is a second one which satisfies $\partial_{t} P=0$ and is given by

$$
P(\{\beta\} ; t)=\prod_{k=1}^{L} p\left(\beta_{k}\right)
$$

where

$$
\begin{aligned}
p(\beta) & =\exp \left(\mu(-1)^{\beta}+\nu\right) \\
\mu & =\ln \sqrt{\frac{w_{1,0}(0,1)}{w_{1,0}(1,1)}}, \quad \nu=-\mu-\ln \left(1+\frac{w_{1,0}(1,1)}{w_{1,0}(0,1)}\right)
\end{aligned}
$$

The probability distribution Eq. (4.42) corresponds to a one-dimensional Ising model defined on the dual lattice and the site variable $\beta_{k}$ corresponds to a link variable of the dual lattice, see also Section 8 .

The existence of two stationary probability distributions satisfying $\partial_{t} P=0$ makes the Hamiltonian $H$ have a degenerate vacuum. The existence of a decoagulation process, no matter how small it is, implies, if the annihilation process is absent, that the stationary configuration should correspond to Eq. (4.42), unless the initial 
state is the empty lattice. If we now turn on the annihilation mechanism, the final configuration will be the empty lattice. Since we always have massive behaviour this transition between stationary states will correspond to a first-order nonequilibrium phase transition which may be accounted by the fact that no symmetry of $H$ is broken. This can now be studied by performing perturbative calculations in the limit of a small decoagulation rate.

We are aware of the following fascinating puzzle. Although the Hamiltonian describing the coagulation and decoagulation processes is not known to be integrable it was shown in [30] that the gap-probability function for this model can be computed exactly and this is an indication that the model is integrable. This is at least the case if one considers the creation-annihilation model with diffusion which will be discussed next (recall that Glauber's solution [19] was discovered without using the JordanWigner transformation). We will return to this problem in a future publication (see also Section 6).

\section{3 $Z_{2}$ and parity invariant, not vacuum-driven processes}

The full master equation without any restrictions includes besides the processes considered so far the following two, see Eq. (4.2)

g.) creation, with rate $w_{1,1}(1,1)$

$$
\emptyset+\emptyset \rightarrow A+A
$$

h.) birth, with rate $w_{1,0}(1,0)$

$$
\emptyset+\emptyset \rightarrow A+\emptyset
$$

From probability conservation, we have

$$
\begin{aligned}
& w_{0,0}(0,0)=w_{1,1}(1,1)+2 w_{1,0}(1,0) \\
& w_{0,0}(1,0)=w_{1,0}(0,0)+w_{1,0}(1,1)+w_{1,1}(1,0) \\
& w_{0,0}(1,1)=2 w_{1,0}(0,1)+w_{1,1}(0,0)
\end{aligned}
$$

The Hamitonian is

$$
\begin{aligned}
H= & \sum_{i=1}^{L-1}\left[\frac{1}{4}\left(w_{0,0}(0,0)+2 w_{0,0}(1,0)+w_{0,0}(1,1)\right)\right. \\
& +\frac{1}{4}\left(w_{0,0}(0,0)+w_{0,0}(1,1)-2 w_{0,0}(1,0)\right) \sigma_{i}^{z} \sigma_{i+1}^{z} \\
& +\frac{1}{4}\left(w_{0,0}(0,0)-w_{0,0}(1,1)\right)\left(\sigma_{i}^{z}+\sigma_{i+1}^{z}\right) \\
& -w_{1,1}(1,0)\left(E_{i}^{01} E_{i+1}^{10}+E_{i}^{10} E_{i+1}^{01}\right) \\
& -\lambda^{2} w_{1,1}(0,0) E_{i}^{01} E_{i+1}^{01}-\lambda^{-2} w_{1,1}(1,1) E_{i}^{10} E_{i+1}^{10}
\end{aligned}
$$




$$
\begin{aligned}
& -\lambda w_{1,0}(0,0)\left(E_{i}^{01} E_{i+1}^{00}+E_{i}^{00} E_{i+1}^{01}\right) \\
& -\lambda^{-1} w_{1,0}(1,0)\left(E_{i}^{10} E_{i+1}^{00}+E_{i}^{00} E_{i+1}^{10}\right) \\
& -\lambda w_{1,0}(0,1)\left(E_{i}^{01} E_{i+1}^{11}+E_{i}^{11} E_{i+1}^{01}\right) \\
& \left.-\lambda^{-1} w_{1,0}(1,1)\left(E_{i}^{10} E_{i+1}^{11}+E_{i}^{11} E_{i+1}^{10}\right)\right],
\end{aligned}
$$

where we have choosen $\frac{h^{(k)}(1)}{h^{(k)}(0)}=\lambda,(k=1,2, \ldots, L)$ in Eq. (2.11). This Hamiltonian has an interesting property. It can always be brought to a hermitian form through a similarity transformation if one out of the three possible forward-backward reactions (annihilation-creation, death-birth, coagulation-decoagulation) take place. If, however, the following relation between the rates is satisfied

$$
\frac{w_{1,1}(1,1)}{w_{1,1}(0,0)}=\left(\frac{w_{1,0}(1,1)}{w_{1,0}(0,1)}\right)^{2}=\left(\frac{w_{1,0}(1,0)}{w_{1,0}(0,0)}\right)^{2}
$$

$H$ can be made hermitian even when all three pairs of reactions occur. The physical significance of Eq. (4.48) has still to be explored.

We shall confine ourselves to the $Z_{2}$ preserving processes which include besides diffusion only annihilation and creation processes. We make the transformation

$$
\frac{\varphi^{(k)}(1,1)}{\varphi^{(k)}(0,0)}=\sqrt{\frac{w_{1,1}(1,1)}{w_{1,1}(0,0)}}, \quad(k=1,2, \ldots, L-1),
$$

and find $H / w_{1,1}(1,0)=H_{0}+H_{1}$ where

$$
\begin{gathered}
H_{0}=-\frac{1}{2} \sum_{i=1}^{L-1}\left[\sigma_{i}^{x} \sigma_{i+1}^{x}+\sigma_{i}^{y} \sigma_{i+1}^{y}+\Delta \sigma_{i}^{z} \sigma_{i+1}^{z}+\left(1-\Delta^{\prime}\right)\left(\sigma_{i}^{z}+\sigma_{i+1}^{z}\right)+\Delta-2\right] \\
H_{1}=-\frac{1}{2} \sum_{i=1}^{L-1} \sqrt{\left(\Delta^{\prime}-\Delta\right)\left(2-\Delta-\Delta^{\prime}\right)}\left(\sigma_{i}^{x} \sigma_{i+1}^{x}-\sigma_{i}^{y} \sigma_{i+1}^{y}\right) \\
\Delta^{\prime}=1-\frac{w_{1,1}(0,0)-w_{1,1}(1,1)}{2 w_{1,1}(1,0)} \\
\Delta=\Delta^{\prime}-\frac{w_{1,1}(1,1)}{w_{1,1}(1,0)}
\end{gathered}
$$

This is just the Hamiltonian corresponding to the kinetic Ising model with purely relaxational dynamics [31 and $H$ is hermitian. The limit when the elements of $H_{1}$ are small does need some care. If the annihilation and creation rates are equal, $\Delta^{\prime}=1$ which allows us to do perturbation theory in the coupling $1-\Delta$ of Eq. (4.50). However, if the rates are not equal and one just takes $w_{1,1}(1,1)$ to zero, the limit for the eigenvectors is singular as is the transformation Eq. (4.49), although the eigenvalue 
spectrum is not affected. Therefore, the heuristically appealing relationship [32] between the kinetic Ising model with Glauber dynamics and the annihilation process should be considered with care if one works (like in Ref. [31]) with the hermitian formulation given by Eq. (4.50).

\section{Exact solution of a two-state system}

In this section, we shall discuss the dynamics of the two-states system described by the Hamiltonian Eq. (4.12) in the free fermion case $\Delta=0$. (For a connexion between this Hamiltonian and a seven-vertex model, see Appendix B.) This model describes $A$ particles diffusing and annihilating in pairs. The motivation for treating this particular case in great detail is twofold.

Firstly, we would like to show that a finite-size scaling theory can be formulated for nonequilibrium systems. This point is particularly important if (in contradistinction to this example) the problem is not exactly solvable. In that case, one will have to solve the problem numerically for finite chains and then extrapolate the results to infinite systems, as one does for equilibrium problems 33 (see also Appendix A). One expects that the finite-size scaling proven for this model is of general validity.

For instance, the concentration of particles per site $\langle n\rangle / L$ obeys the relation

$$
c=L^{x} \phi(z)
$$

where $z=4 D t / L^{2}$ and $\phi(z) \sim z^{-\alpha}$ as $z \rightarrow 0$. Moreover, $x$ is related to the large time behaviour of $c \sim t^{-\alpha}$, namely $x=-2 \alpha$.

Secondly, we want to stress the interest of the (connected) two-point function

$$
G(r, t)=<n_{m} n_{m+r}>-<n_{m}><n_{m+r}>
$$

where $m$ indicates the position of a lattice site. Here, we shall pose two different questions about $G(r, t)$. First, we ask for the large time behaviour $(t \rightarrow \infty)$ if $r$ is kept fixed and look for the exponent $v_{r}$

$$
G(r, t) \sim t^{v_{r}}
$$

Secondly, we consider the scaling limit where simultaneously $r, t \rightarrow \infty$ such that $u=r^{2} / t$ is fixed and define a critical exponent $y$

$$
G(r, t) \sim r^{-y} g(u)
$$

where $g$ is a scaling function. We believe that for nonequilibrium problems the exponents $y$ 's might turn out to be as fundamental as the critical exponents of twodimensional equilibrium statistical mechanics, since the information given by the initial probability function should be hidden in the scaling function $g(u)$ and consequently the $y^{\prime}$ 's should be universal (see also Ref. [32]). 
Finally, we would like to present powerful techniques which allow us to reduce the calculation of nonequilibrium averages, see eq. (3.18), to vacuum expectation values, thereby extending the approach initiated by Lushnikov [34] to the calculation of correlation functions.

The quantum Hamiltonian $H$ (4.12) contains the parameter

$$
\epsilon=\frac{1}{2}\left(q+q^{-1}\right)-1 \geq 0
$$

where $\epsilon$ measures the left-right asymmetry of the diffusion process. In the remainder of this section we take $\epsilon=0$. We do not make use of the change of variables given by the function $\varphi^{(i)}(\alpha, \beta)$ described in the last section. In terms of Pauli matrices $\sigma^{ \pm}=\left(\sigma^{x} \pm i \sigma^{y}\right) / 2$, the Hamiltonian is equivalent to

$$
H=-D \sum_{n=1}^{L}\left[\sigma_{n}^{+} \sigma_{n+1}^{-}+\sigma_{n}^{-} \sigma_{n+1}^{+}-2 \sigma_{n}^{+} \sigma_{n}^{-}+2 \sigma_{n}^{-} \sigma_{n+1}^{-}\right]
$$

where $D$ is the diffusion constant. In this section for simplicity we only use periodic boundary conditions.

\subsection{Diagonalization and the generating function}

The diagonalization $H$ is fairly standard. By restricting ourselves to the case $L=2 M$ even we define fermionic variables via the Jordan-Wigner transformation

$$
\begin{aligned}
\sigma_{n}^{-} & =(-1)^{\sum_{m<n} a_{m}^{+} a_{m}} a_{n} \\
\sigma_{n}^{+} & =a_{n}^{+}(-1)^{\sum_{m<n} a_{m}^{+} a_{m}}
\end{aligned}
$$

as well as their Fourier transforms

$$
\begin{aligned}
& a_{n}=L^{-1 / 2} e^{-i \pi / 4} \sum_{q} e^{i q n} \tilde{a}_{q} \\
& a_{n}^{+}=L^{-1 / 2} e^{+i \pi / 4} \sum_{q} e^{-i q n} \tilde{a}_{q}^{+}
\end{aligned}
$$

In terms of these fermionic variables the Hamiltonian Eq. (5.6) takes the form

$$
\begin{aligned}
H= & -2 D \sum_{0 \leq q<\pi}\left[(\cos q-1)\left(\tilde{a}_{q}^{+} \tilde{a}_{q}+\tilde{a}_{-q}^{+} \tilde{a}_{-q}\right)+2 \sin q \tilde{a}_{q} \tilde{a}_{-q}\right. \\
& \left.-\delta_{q, \pi} 2 \tilde{a}_{\pi}^{+} \tilde{a}_{\pi}\right]
\end{aligned}
$$

where $q$ takes the values

$$
\begin{aligned}
q_{\text {even }} & =\frac{2 k-1}{2 M} \pi, k=1,2, \ldots, M \\
q_{\text {odd }} & =\frac{2 k}{2 M} \pi, k=0,1, \ldots, M
\end{aligned}
$$


if the total number of fermions $\mathcal{N}=\sum_{m=1}^{L} a_{m}^{+} a_{m}$ is an even or odd number, respectively. The Hilbert space associated to Eq. (5.9) can therefore be splitted into two disjoints sectors depending on the parity of the fermion number. The sector with $\mathcal{N}$ odd $(\mathcal{N}$ even) will be related to the case where the ground state has a single particle (no particle).

Looking again at Eq. (5.9), we note that $H=\sum_{0 \leq q \leq \pi} H_{q}$ has block-diagonal structure. The time-evolution equation for wave functions $\partial_{t} \Phi=-H \Phi$ can be broken up into separate equations for each block

$$
\Phi(t)=\prod_{0 \leq q \leq \pi} \Phi_{q}(t), \partial_{t} \Phi_{q}=-H_{q} \Phi_{q} ;(0 \leq q \leq \pi)
$$

Each block is generated by acting with $\tilde{a}_{q}^{+}$and $\tilde{a}_{-q}^{+}$on the state $|v a c\rangle$ without fermions, i.e. $\tilde{a}_{q}|v a c\rangle=0$. If $q \neq 0, \pi, H_{q}$ can be written as a $4 \times 4$ matrix. In the basis $|0\rangle=|v a c\rangle,|1\rangle=\tilde{a}_{q}^{+}|v a c\rangle,|2\rangle=\tilde{a}_{-q}^{+}|v a c\rangle$ and $|3\rangle=\tilde{a}_{q}^{+} \tilde{a}_{-q}^{+}|v a c\rangle$ it takes the form

$$
H_{q}=-2 D\left(\begin{array}{cccc}
0 & & & -2 \sin q \\
& \cos q-1 & & \\
& & \cos q-1 & \\
& & & 2 \cos q-2
\end{array}\right)
$$

while for $q=0, \pi$, the blocks $H_{0}$ and $H_{\pi}$ are already diagonal. The general solution of Eq. (5.11) corresponds to

$$
\Phi_{q}(t)=\left(\alpha_{q}(t) \tilde{a}_{q}^{+} \tilde{a}_{-q}^{+}+\gamma_{q}(t) \tilde{a}_{q}^{+}+\delta_{q}(t) \tilde{a}_{-q}^{+}+\beta_{q}(t)\right)|v a c\rangle
$$

where the functions $\alpha, \beta, \gamma, \delta$ satisfy the differential equations

$$
\begin{aligned}
\dot{\alpha}_{q}(t) & =4 D(\cos q-1) \alpha_{q}(t) \\
\dot{\beta}_{q}(t) & =-4 D \sin q \alpha_{q}(t) \\
\dot{\gamma}_{q}(t) & =2 D(\cos q-1) \gamma_{q}(t) \\
\dot{\delta}_{q}(t) & =2 D(\cos q-1) \delta_{q}(t)
\end{aligned}
$$

The solutions of these equations are promptly derived

$$
\begin{aligned}
\alpha_{q}(t) & =\alpha_{q}(0) \exp [-4 D t(1-\cos q)] \\
\beta_{q}(t) & =\beta_{q}(0)+\cot \frac{q}{2}\left\{\alpha_{q}(t)-\alpha_{q}(0)\right\} \\
\gamma_{q}(t) & =\gamma_{q}(0) \exp [-2 D t(1-\cos q)] \\
\delta_{q}(t) & =\delta_{q}(0) \exp [-2 D t(1-\cos q)]
\end{aligned}
$$

For an arbitrary initial condition the general solution will be given by linear combinations of the wave functions Eq. (5.11). A general study considering arbitrary general initial conditions is not straightforward and is not in our intentions. Since our main interest here is the finite-size scaling behaviour of the system, we will hereafter choose 
the simple initial condition where we have no vacancies present. This condition was used by Lushnikov [34 and corresponds to the situations where we have a single wave function (5.11), i.e. $\psi(t)=\Phi(t)$ with

$$
\alpha_{q}(0)=-1, \beta_{q}(0)=\gamma_{q}(0)=\delta_{q}(0)=0,
$$

where $q$ takes the values $q_{\text {even }}$ in the set (5.10), since in this case the fermion number $\mathcal{N}$ is even.

In order to calculate the concentration of particles and correlations, following the work of Lushnikov [34] it is convenient to introduce generating functions. Since (see Section 3) the wave function takes the role of the probability distribution introduced earlier, a generating function convenient for our purpose is defined by

$$
F(\{z\}, t)=F\left(z_{1}, \ldots, z_{L}, t\right)=\frac{\sum_{\{\mathcal{C}\}} \prod_{i=1}^{L} z_{i}^{n_{i}} \psi(\{\mathcal{C}\} ; t)}{\sum_{\{\mathcal{C}\}} \psi(\{\mathcal{C}\} ; t)}
$$

where $n_{i}=0,1$ and $z_{i}$ denote the occupation number and fugacity at the site $i, \mathcal{C}$ is a configuration $\left\{n_{1}, \ldots, n_{L}\right\}$ of occupied or empty sites. In Eq. (5.17) we take into account that $\psi$ does not, in general, satisfy the normalization condition of a probability distribution. Using the results of Section 3, in particular (3.14), we can bring the calculation of $F(\{z\}, t)$ to an equilibrium problem, i.e.

$$
\begin{aligned}
F(\{z\}, t) & =F_{0}(t) \sum_{\{\mathcal{C}\}} \prod_{i=1}^{L} z_{i}^{n_{i}} \psi(\{\mathcal{C}\} ; t) \\
& =F_{0}(t)\left\langle v a c\left|\exp \left(\sum_{\ell=1}^{L} z_{\ell} \sigma^{-}(\ell)\right) \prod_{q>0}\left(\alpha_{q}(t) \tilde{a}_{q}^{+} \tilde{a}_{-q}^{+}+\beta_{q}(t)\right)\right| v a c\right\rangle(5 .
\end{aligned}
$$

where we have already used the initial condition Eq. (5.16) and $F_{0}(t)$ is determined from the normalization condition $F(1, \ldots, 1, t)=1$ for all $t$. In order to understand Eq. (5.19), it is useful to consider the identity

$$
\exp \left(\sum_{m=1}^{L} z_{m} \sigma^{-}(m)\right)=\sum_{k=0}^{L} \sum_{m_{1}>m_{2}>\ldots>m_{k}} z_{m_{1}} \sigma^{-}\left(m_{1}\right) \cdots z_{m_{k}} \sigma^{-}\left(m_{k}\right)
$$

which we can derive by expanding the exponentials and using the relations $\left(\sigma^{-}(m)\right)^{2}=$ 0 and $\left[\sigma^{-}(m), \sigma^{-}\left(m^{\prime}\right)\right]=0$. It is now clear that $\langle v a c| \exp \left(\sum_{\ell=1}^{L} z_{\ell} \sigma^{-}(\ell)\right)$ corresponds to the state $\langle 0|$ of Section 3 .

In order to calculate $F(\{z\}, t)$ we observe that due to the ordering $m_{1}>m_{2}>$ $\ldots>m_{k}$ we get

$$
\langle v a c| \sigma^{-}\left(m_{1}\right) \sigma^{-}\left(m_{2}\right) \ldots \sigma^{-}\left(m_{k}\right)=\langle v a c| a_{m_{1}} a_{m_{2}} \ldots a_{m_{k}}
$$

On the other hand, we also have from Eq. (5.8)

$$
\tilde{a}_{q}^{+} \tilde{a}_{-q}^{+}=\frac{2}{L} \sum_{m} \sum_{n>m} \sin (q(n-m)) a_{n}^{+} a_{m}^{+}
$$


Because of the product structure Eqs. (5.11, 5.13) of $\phi$, the generating function can also be written as $F=\prod_{q>0} F_{q}$. Each factor contains only two fermionic creation operators at most. So we only need to calculate

$$
\begin{aligned}
& \sum_{m^{\prime}} \sum_{m>m^{\prime}}\left\langle v a c\left|\sigma^{-}(m) \sigma^{-}\left(m^{\prime}\right) \tilde{a}_{q}^{+} \tilde{a}_{-q}^{+}\right| v a c\right\rangle \\
& =\frac{2}{L} \sum_{n^{\prime}, m^{\prime}} \sum_{m>m^{\prime}} \sum_{n>n^{\prime}}\left[\sin \left(q\left(n-n^{\prime}\right)\right)\left\langle v a c\left|a_{m} a_{m^{\prime}} a_{n}^{+} a_{n^{\prime}}^{+}\right| v a c\right\rangle\right] \\
& =\frac{2}{L} \sum_{n^{\prime}, m^{\prime}} \sum_{m>m^{\prime}} \sum_{n>n^{\prime}}\left[\sin \left(q\left(n-n^{\prime}\right)\right)\left(\delta_{n, m^{\prime}} \delta_{n^{\prime}, m}-\delta_{n, m} \delta_{n^{\prime}, m^{\prime}}\right)\right] \\
& =-\frac{2}{L} \sum_{n^{\prime}} \sum_{n>n^{\prime}} \sin \left(q\left(n-n^{\prime}\right)\right)
\end{aligned}
$$

In the sequel, we shall need two identities, which are obtained using Eq. (5.10), with $m$ fixed

$$
\begin{aligned}
\sum_{n>m} \sin (q(n-m)) & =\frac{1}{2}\left[\cot \frac{q}{2}+\frac{\cos (q(m-1 / 2))}{\sin (q / 2)}\right] \\
\sum_{n<m} \sin (q(m-n)) & =\frac{1}{2}\left[\cot \frac{q}{2}-\frac{\cos (q(m-1 / 2))}{\sin (q / 2)}\right]
\end{aligned}
$$

We now combine Eqs. (5.18) - (5.24) and get

$$
F(\{z\}, t)=F_{0}(t) \prod_{q>0}\left(\alpha_{q}(t) \cdot\left(-\frac{2}{L} \sum_{m} \sum_{n>m} z_{n} z_{m} \sin (q(n-m))\right)+\beta_{q}(t)\right)
$$

We fix $F_{0}(t)$ from the condition $F(1, \ldots, 1, t)=1$ for all $t$. The final result for the generating function is

$$
F(\{z\}, t)=\prod_{q>0}\left(\frac{\alpha_{q}(t) \cdot\left(-\frac{2}{L} \sum_{m} \sum_{n>m} z_{n} z_{m} \sin (q(n-m))\right)+\beta_{q}(t)}{-\alpha_{q}(t) \cot \frac{q}{2}+\beta_{q}(t)}\right)
$$

\subsection{Calculation of the mean concentration}

The mean number of particles at an arbitrary site $m$ for a chain of length $L$ is now obtained from

$$
<n_{m}>=\left.z_{m} \frac{\partial}{\partial z_{m}} F(\{z\}, t)\right|_{z_{1}=\ldots=z_{L}=1}=L^{-1} \sum_{q>0}\left(\frac{-2 \alpha_{q}(t) \cot \frac{q}{2}}{-\alpha_{q}(t) \cot \frac{q}{2}+\beta_{q}(t)}\right)
$$

Due to the translational invariance of the particular initial state considered here (Eq. (5.16)) the concentration of particles per site is simply

$$
c=<n_{m}>
$$

Substituting Eqs. (5.13, 5.16) into (5.27) we obtain

$$
c(t)=2 L^{-1} \sum_{q>0} \exp (-4 D t(1-\cos q))
$$




\subsection{Large-time behaviour and finite-size scaling}

We now discuss the late stages dependence of the concentration $c(t)$. In the limit $L \rightarrow \infty$ for $t$ fixed

$$
c(t)=\frac{1}{\pi} \int_{0}^{\pi} e^{-4 D t(1-\cos q)} d q=e^{-4 D t} I_{0}(4 D t)
$$

where $I_{0}(x)$ is a modified Bessel function. For $D t \gg 1$ one gets

$$
c(t) \simeq(8 \pi D t)^{-1 / 2}
$$

Let us now analyse the regime of finite-size scaling where $L \rightarrow \infty, t \rightarrow \infty$ but $t L^{-2}$ fixed. In this case we obtain

$$
\begin{aligned}
c & =\frac{2}{L} \sum_{k=1}^{\infty} \exp \left(-2 \pi^{2} z\left(k-\frac{1}{2}\right)^{2}\right) \\
& =\frac{1}{L} \Theta_{2}(0,2 \pi i z) \\
& =\frac{1}{L} \sqrt{\frac{1}{2 \pi z}}\left(1+2 \sum_{\ell=1}^{\infty}(-1)^{\ell} \exp \left(-\ell^{2} /(2 z)\right)\right)
\end{aligned}
$$

where $\Theta_{2}(z, \tau)$ is a Jacobi theta function and

$$
z=4 D t L^{-2}
$$

is the finite-size scaling variable. Eq. (5.34) is obtained from (5.32) by using the Poisson resummation formula. From Eqs. (5.32-5.34) and (5.1) we get the critical exponent $x=-1$.

\subsection{Fluctuations around the mean values}

Next, we briefly discuss the fluctuations around $\left\langle n_{m}>\right.$. For simplicity, we take $z_{1}=\ldots=z_{L}=z$ in Eq. (5.17). Then the generating function $F=F(z, t)$ and we have

$$
\begin{aligned}
<n_{m}^{2}> & =\left.\frac{1}{L^{2}}\left(z \frac{\partial}{\partial z}\right)^{2} F(z, t)\right|_{z=1} \\
& =<n_{m}>^{2}+2<n_{m}>/ L-\frac{1}{L^{2}} \sum_{q>0}\left(\frac{-2 \alpha_{q}(t) \cot \frac{q}{2}}{-\alpha_{q}(t) \cot \frac{q}{2}+\beta_{q}(t)}\right)^{2}
\end{aligned}
$$

We get

$$
\begin{aligned}
<n_{m}^{2}>-<n_{m}>^{2} & =2 / L\left(e^{-4 D t} I_{0}(4 D t)-e^{-8 D t} I_{0}(8 D t)\right) \\
& \simeq \frac{1}{L} \frac{1}{\sqrt{2 \pi D t}}\left(1-\frac{1}{\sqrt{2}}\right)
\end{aligned}
$$


as $t \rightarrow \infty$. This means that for fixed, but large $t$

$$
<\delta n_{m}^{2}>/<n_{m}>^{2} \sim t^{1 / 2} L^{-1}
$$

which accounts for the fact that as the time grows the annihilation of couples of particles induces larger fluctuations in the particle concentration.

\subsection{Correlation functions}

We now turn towards the calculation of correlation functions. As an example which illustrates the general technique, we calculate the connected correlation function defined in Eq. (5.2). From the definition of the generating function (5.17) we can write

$$
G(r, t)=<n_{m} n_{m+r}>-<n_{m}>^{2}=\left.\frac{\partial^{2}}{\partial z_{m} \partial z_{m+r}} \ln F(\{z\}, t)\right|_{z_{1}=\ldots=z_{L}=1}
$$

A straightforward calculation gives us

$$
G(r, t)=\frac{2}{L} \sum_{q>0}\left(\frac{-\sin (q r) \alpha_{q}(t)}{-\alpha_{q}(t) \cot \frac{q}{2}+\beta_{q}(t)}\right)-\frac{4}{L^{2}} \sum_{q>0}\left(\frac{-\alpha_{q}(t) \cot \frac{q}{2}}{-\alpha_{q}(t) \cot \frac{q}{2}+\beta_{q}(t)}\right)^{2}
$$

which does not depend on $m$ due to the fact that the Hamiltonian Eq. (5.6) as well as the initial probability distribution are translational invariant.

Analysing the relative importance of the two terms contributing to $G(r, t)$, we see that in the limit $L \rightarrow \infty$, the first term is of order unity, while the second one is of order $\mathcal{O}\left(L^{-1}\right)$.

This correlation can be evaluated in the same way as we did for the concentration. Let us consider initially the case of the correlation of next-neighbour particles. We find

$$
\begin{aligned}
G(1, t) & =\frac{2}{L} \sum_{q>0}(1-\cos q) \exp [-4 D t(1-\cos q)] \\
& =e^{-4 D t}\left[I_{0}(4 D t)-I_{1}(4 D t)\right] \\
& \simeq \pi(8 \pi D t)^{-3 / 2}
\end{aligned}
$$

as $t \rightarrow \infty$. The same result can be obtained using Glauber's dynamics for the Ising model at $T=0$ (see also Section 8) and considering domain walls two-point functions [35]. The initial condition we are considering in the diffusion-annihilation problem (no vacancies) corresponds to a fully ordered antiferromagnetic state in the kinetic Ising model. On the other hand, for arbitrary but finite $r$, we obtain analogously

$$
G(r, t) \simeq r G(1, t)
$$

This result reflects the fact that at late times it is more difficult to find particles close to each other than far apart. From Eq. (5.3) this gives us the exponents $v_{r}=-3 / 2$. 
Finally, in connection with Eq. (5.4), let us consider the limit where $r \rightarrow \infty$, $t \rightarrow \infty$ but $u=r^{2} / t$ stays finite. This leads to

$$
G \simeq \pi(8 \pi D)^{-3 / 2} r^{-2} u^{3 / 2} e^{-u /(2 D)}
$$

and we read off the exponent $y=-2$ and recognize the scaling function $g(u)=$ $\pi(8 \pi D)^{-3 / 2} u^{3 / 2} e^{-u /(2 D)}$, see Eq. (5.4). We remark that if we consider the domain walls two-point function in the zero-temperature kinetic Ising model with Glauber dynamics [35] for an initial probability distribution with zero magnetization (which is a different initial condition than that we are considering here), we obtain the same result. This is an indication in favour of our conjecture that the $y$ exponents are universal.

\section{Three-state models with $Z_{3}$ symmetry and va- cuum-driven processes}

As noticed in Section 4 , behind many processes in the two-states models there is the XXZ model in a magnetic field. We shall now show that there are several integrable quantum chains which play a similar role for three-states models with $Z_{3}$ symmetry. We shall restrict ourselves to the $Z_{3}$-symmetric case for simplicity. This already contains the two-species annihilation process but not the $Z_{2}$ symmetric trapping

reaction to which we hope to return in a future publication. Let 0 stand for a vacancy, 1 for the particle $A$ and 2 for the particle $B$.

We use the general results of Section 3 and the conditions Eqs. (2.5,2.12) and derive the Hamiltonian

$$
\begin{aligned}
& H=U-T \\
& T=\sum_{i=1}^{L-1}\left[w_{2,1}(0,0) E_{i}^{02} E_{i+1}^{01}+w_{1,2}(0,0) E_{i}^{01} E_{i+1}^{02}\right. \\
&+w_{2,1}(1,0) E_{i}^{10} E_{i+1}^{01}+w_{1,2}(0,1) E_{i}^{01} E_{i+1}^{10} \\
& \quad+w_{2,1}(2,1) E_{i}^{21} E_{i+1}^{12}+w_{1,2}(1,2) E_{i}^{12} E_{i+1}^{21} \\
& \quad+w_{2,1}(0,2) E_{i}^{02} E_{i+1}^{20}+w_{1,2}(2,0) E_{i}^{20} E_{i+1}^{02} \\
&+w_{2,1}(1,1) E_{i}^{10} E_{i+1}^{12}+w_{1,2}(0,2) E_{i}^{01} E_{i+1}^{21} \\
&+w_{2,1}(2,0) E_{i}^{21} E_{i+1}^{01}+w_{1,2}(1,1) E_{i}^{12} E_{i+1}^{10} \\
&+w_{2,1}(0,1) E_{i}^{02} E_{i+1}^{12}+w_{1,2}(2,2) E_{i}^{20} E_{i+1}^{21} \\
&+w_{2,1}(2,2) E_{i}^{21} E_{i+1}^{20}+w_{1,2}(1,0) E_{i}^{12} E_{i+1}^{02} \\
&\left.+w_{2,1}(1,2) E_{i}^{10} E_{i+1}^{20}+w_{1,2}(2,1) E_{i}^{20} E_{i+1}^{10}\right] \\
& \sum_{i=1}^{L-1}\left[\left(w_{2,1}(2,0)+w_{1,2}(0,2)\right) E_{i}^{11} E_{i+1}^{11}+\left(w_{2,1}(0,1)+w_{1,2}(1,0)\right) E_{i}^{22} E_{i+1}^{22}\right. \\
&
\end{aligned}
$$




$$
\begin{aligned}
& +\left(w_{2,1}(1,0)+w_{1,2}(2,2)\right) E_{i}^{00} E_{i+1}^{11}+\left(w_{2,1}(2,2)+w_{1,2}(0,1)\right) E_{i}^{11} E_{i+1}^{00} \\
& +\left(w_{2,1}(1,1)+w_{1,2}(2,0)\right) E_{i}^{00} E_{i+1}^{22}+\left(w_{2,1}(0,2)+w_{1,2}(1,1)\right) E_{i}^{22} E_{i+1}^{00} \\
& +\left(w_{2,1}(2,1)+w_{1,2}(0,0)\right) E_{i}^{11} E_{i+1}^{22}+\left(w_{2,1}(0,0)+w_{1,2}(1,2)\right) E_{i}^{22} E_{i+1}^{11} \\
& \left.+\left(w_{2,1}(1,2)+w_{1,2}(2,1)\right) E_{i}^{00} E_{i+1}^{00}\right]
\end{aligned}
$$

Notice that for the time being we do not use any equivalence transformation (2.8) as we did in Sec.(4).

We first consider the case of asymmetric diffusion

$$
\left\{\begin{array}{cl}
\emptyset+A \rightarrow A+\emptyset & \text { with rate } w_{2,1}(1,0) \\
A+B \rightarrow B+A & \text { with rate } w_{2,1}(2,1) \\
B+\emptyset \rightarrow \emptyset+B & \text { with rate } w_{2,1}(0,2) \\
A+\emptyset \rightarrow \emptyset+A & \text { with rate } w_{1,2}(0,1) \\
B+A \rightarrow A+B & \text { with rate } w_{1,2}(1,2) \\
\emptyset+B \rightarrow B+\emptyset & \text { with rate } w_{1,2}(2,0)
\end{array}\right.
$$

and let us assume that we have

$$
\begin{aligned}
& w_{2,1}(1,0)=w_{1,2}(2,0)=w_{2,1}(2,1)=\Gamma_{L} \\
& w_{1,2}(0,1)=w_{2,1}(0,2)=w_{1,2}(1,2)=\Gamma_{R} .
\end{aligned}
$$

By defining

$$
q=\sqrt{\frac{\Gamma_{L}}{\Gamma_{R}}}
$$

we now obtain

$$
\begin{aligned}
H= & D\left\{\sum _ { i = 1 } ^ { L - 1 } \left[\frac{q+q^{-1}}{2}-\left(q \sum_{b>a} E_{i}^{a b} E_{i+1}^{b a}+q^{-1} \sum_{b<a} E_{i}^{a b} E_{i+1}^{b a}\right.\right.\right. \\
& \left.\left.\left.\frac{q+q^{-1}}{2} \sum_{a=0}^{2} E_{i}^{a a} E_{i+1}^{a a}+\frac{q-q^{-1}}{2} \sum_{a \neq b} \operatorname{sign}(a-b) E_{i}^{a a} E_{i+1}^{b b}\right)\right]\right\}
\end{aligned}
$$

where $D=\sqrt{\Gamma_{L} \Gamma_{R}}$ is the diffusion constant. Through a similarity transformation $S$, we can bring this Hamiltonian to the form

$$
\begin{aligned}
H^{\prime}=S H S^{-1}= & D\left\{\sum _ { i = 1 } ^ { L - 1 } \left[\frac{q+q^{-1}}{2}-\left(\sum_{a \neq b} E_{i}^{a b} E_{i+1}^{b a}+\frac{q+q^{-1}}{2} \sum_{a=0}^{2} E_{i}^{a a} E_{i+1}^{a a}\right.\right.\right. \\
& \left.\left.\left.+\frac{q-q^{-1}}{2} \sum_{a \neq b} \operatorname{sign}(a-b) E_{i}^{a a} E_{i+1}^{b b}\right)\right]\right\}
\end{aligned}
$$

This similarity transformation does not have the simple local form of Eq. (2.8). The argument goes as follows. We first notice that the Hamiltonian $H^{\prime}$ given by Eq.(6.8) is the exactly integrable $U_{q} S U(3)$ chain [36] and corresponds to the anisotropic version 
of the spin 1 model introduced by Sutherland [37], (see also Appendix C, where the connexion with the Hecke algebra is shown). On the other hand $H^{\prime}$ can be brought by an equivalent transformation to the four-parameter deformation of the $S U(3)$ symmetric chain 38]

$$
\begin{aligned}
H^{\prime \prime}= & \tilde{S} H^{\prime} \tilde{S}^{-1}=D\left\{\sum _ { i = 1 } ^ { L - 1 } \left[\frac{q+q^{-1}}{2}-\left(f_{10} E_{i}^{10} E_{i+1}^{01}+f_{10}^{-1} E_{i}^{01} E_{i+1}^{10}\right.\right.\right. \\
& +f_{20} E_{i}^{20} E_{i+1}^{02}+f_{20}^{-1} E_{i}^{02} E_{i+1}^{20}+f_{21} E_{i}^{21} E_{i+1}^{12}+f_{21}^{-1} E_{i}^{12} E_{i+1}^{21} \\
& \left.\left.\left.+\frac{q+q^{-1}}{2} \sum_{a=0}^{2} E_{i}^{a a} E_{i+1}^{a a}+\frac{q-q^{-1}}{2} \sum_{a \neq b} \operatorname{sign}(a-b) E_{i}^{a a} E_{i+1}^{b b}\right)\right]\right\}
\end{aligned}
$$

We observe that if in Eq.(6.9) we take $f_{21}=f_{31}=f_{32}=q$ we recover Eq.(6.8). The equivalence between the chain given by Eq. (6.7) and the $U_{q} S U(3)$ chain (6.8) is similar to the two-state model in the case of an asymmetric definition, where we got $U_{q} S U(2)$. The chain Eq. (6.8) is massive unless $q=1$, which corresponds to the left-right symmetric case and the physical interpretation for these results is similar to the two species case of Section 4 . The case where the process $A+B \rightarrow B+A$ is suppressed is, as shown in Appendix $C$, again related to a quotient of a Hecke algebra and the eigenvalues of the Hamiltonian are the same (the degeneracies may be different) as for the $U_{q} S U(2)$ symmetric one given by Eq. (4.17).

We shall take from now on $q=1$ and parity conservation. We assume that the diffusion rates are

$$
\begin{aligned}
& w_{2,1}(1,0)=w_{1,2}(0,1)=w_{2,1}(0,2)=w_{1,2}(2,0)=D \\
& w_{2,1}(2,1)=w_{1,2}(1,2)=\lambda D
\end{aligned}
$$

We consider now the annihilation processes

$$
\begin{cases}A+B \rightarrow \emptyset+\emptyset & \text { with rate } w_{1,2}(0,0)=w_{2,1}(0,0)=\alpha_{A B} D \\ A+A \rightarrow \emptyset+B & \text { with rate } w_{1,2}(0,2)=w_{2,1}(2,0)=\alpha_{A A} D \\ B+B \rightarrow \emptyset+A & \text { with rate } w_{1,2}(1,0)=w_{2,1}(0,1)=\alpha_{B B} D\end{cases}
$$

In this case, the Hamiltonian has a special form

$$
H=D\left(H_{0}+H_{1}\right)
$$

where

$$
\begin{aligned}
H_{0}= & \sum_{i=1}^{L-1}\left[2 \alpha_{A A} E_{i}^{11} E_{i+1}^{11}+2 \alpha_{B B} E_{i}^{22} E_{i+1}^{22}\right. \\
& +\left(\lambda+\alpha_{A B}\right)\left(E_{i}^{11} E_{i+1}^{22}+E_{i}^{22} E_{i+1}^{11}\right) \\
& +E_{i}^{00}\left(E_{i+1}^{11}+E_{i+1}^{22}\right)+\left(E_{i}^{11}+E_{i}^{22}\right) E_{i+1}^{00} \\
& -E_{i}^{10} E_{i+1}^{01}-E_{i}^{01} E_{i+1}^{10}-E_{i}^{20} E_{i+1}^{02}-E_{i}^{02} E_{i+1}^{20}
\end{aligned}
$$




$$
\begin{aligned}
& \left.-\lambda\left(E_{i}^{21} E_{i+1}^{12}+E_{i}^{12} E_{i+1}^{21}\right)\right] \\
H_{1}= & -\sum_{i=1}^{L-1}\left[\alpha_{A B}\left(E_{i}^{01} E_{i+1}^{02}+E_{i}^{02} E_{i+1}^{01}\right)\right. \\
& \left.+\alpha_{A A}\left(E_{i}^{21} E_{i+1}^{01}+E_{i}^{01} E_{i+1}^{21}\right)+\alpha_{B B}\left(E_{i}^{02} E_{i+1}^{12}+E_{i}^{12} E_{i+1}^{02}\right)\right]
\end{aligned}
$$

Using the arguments from Section [4, one can check that the spectrum of the hermitian operator $H_{0}$ coincides with the one of $H / D$. This Hamiltonian describes the pure reaction $A+B \rightarrow \emptyset$, if we take

$$
\lambda=\alpha_{A A}=\alpha_{B B}=0
$$

Let us find cases where the Hamiltonian is integrable. First we look at the $U_{q} S U(3)$ chain Eq. (6.8) with $q=-1$. If we add to the Hamitonian fields along the generators of the algebra, the system stays integrable since the supplementary terms commute with the integrable $U_{q} S U(3)$ Hamiltonian. We found only one case where this is possible. Taking

$$
\lambda=1, \quad \alpha_{A A}=\alpha_{A B}=\alpha_{B B}=2
$$

we find

$$
H_{0}=\sum_{i=1}^{L-1}\left[-1+\sum_{a \neq b} E_{i}^{a b} E_{i+1}^{b a}-\sum_{a=0}^{2} E_{i}^{a a} E_{i+1}^{a a}+2\left(\sigma_{i}^{0}+\sigma_{i+1}^{0}\right)\right]
$$

The first three terms in Eq. (6.17) give a $S U(3)$ symmetric Hamiltonian, corresponding to $q=-1$ in Eq. (6.7). The last term with

$$
\sigma^{0}=\left(\begin{array}{ccc}
0 & 0 & 0 \\
0 & 1 & 0 \\
0 & 0 & 1
\end{array}\right)
$$

is an external field. This observation should allow us to study the critical properties of this chemical process.

Another possibility occurs when $\lambda=0$. We can rewrite $H_{0}$ in the following form, assuming $\alpha_{A A}=\alpha_{B B}$

$$
\begin{gathered}
H_{0}=\sum_{i=1}^{L-1}\left[\left(\frac{2 \alpha_{A A}+\alpha_{A B}}{2}-2\right) \sigma_{i}^{0} \sigma_{i+1}^{0}+\left(\frac{2 \alpha_{A A}-\alpha_{A B}}{2}\right) \sigma_{i}^{z} \sigma_{i+1}^{z}\right. \\
\left.+\sigma_{i}^{0}+\sigma_{i+1}^{0}-\tau_{i}^{+} \tau_{i+1}^{-}-\tau_{i}^{-} \tau_{i+1}^{+}-\rho_{i}^{+} \rho_{i+1}^{-}-\rho_{i}^{-} \rho_{i+1}^{+}\right]
\end{gathered}
$$

where we have used the notations

$$
\tau^{+}=E^{01}, \tau^{-}=E^{10}, \rho^{+}=E^{02}, \rho^{-}=E^{20}, \sigma^{z}=E^{11}-E^{22}
$$

This Hamiltonian commutes with $\Sigma^{z}$ and $\Sigma^{0}$ where

$$
\Sigma^{z}=\sum_{i=1}^{L} \sigma_{i}^{z}, \quad \Sigma^{0}=\sum_{i=1}^{L} \sigma_{i}^{0}
$$


For the special choice

$$
\alpha_{A B}=2 \alpha_{A A}
$$

the Hamiltonian has the larger symmetry $S U(2) \otimes U(1)$ since $H_{0}$ commutes also with $\Sigma^{ \pm}$which are defined by

$$
\Sigma^{+}=\sum_{i=1}^{L} \sigma_{i}^{+}, \quad \Sigma^{-}=\sum_{i=1}^{L} \sigma_{i}^{-}
$$

using $\sigma^{+}=E^{12}$ and $\sigma^{-}=E^{21}$.

If we finally take $\alpha_{A A}=1$, we have

$$
H_{0}=\sum_{i=1}^{L-1}\left[\left(\sigma_{i}^{0}+\sigma_{i+1}^{0}\right)-\tau_{i}^{+} \tau_{i+1}^{-}-\tau_{i}^{-} \tau_{i+1}^{+}-\rho_{i}^{+} \rho_{i+1}^{-}-\rho_{i}^{-} \rho_{i+1}^{+}\right]
$$

The chain Eq. (6.24) is integrable and massless as shown in Appendix C. It can also be related to $t-J$ model [51] with $J=0$. Moreover the spectrum of $H_{0}$ (not the degeneracies) is that of a free fermion system. In Appendix $\mathrm{C}$ it is also shown that not only $H_{0}$ but also $H$ can be expressed in terms of a Hecke algebra which implies that the system is integrable. This remains valid even if we don't assume left-right symmetry. The quantum chain given by Eq. 6.24) was discovered independently in Refs. [53] and [54]. In the first reference this chain was found as a special case in a search of chains related to Hecke algebras. In the second reference it corresponds to a special case of integrable chains defined on nilpotent representations of the $U_{q} S U(2)$ quantum group with $q=\exp \frac{2 \pi i}{3}$. In the last reference the $S U(2) \otimes U(1)$ symmetry was observed. This triggered a further investigation by two of the authors to find the whole symmetry of the chain and obtain its eigenspectrum [55]. We would like to mention that for this system an exact result concerning the time-dependence of the total concentration $\left\langle c_{A}+c_{B}\right\rangle$ is already known [39]. Moreover, if we keep the relation (6.22) but let the parameter $\alpha_{A A}$ free, one can show that the spectrum (not the degeneracies) is the same as that of the XXZ model in a field, see Eq. (4.20) with $\Delta=1-\alpha_{A A}$ and $h=1-\Delta$, which corresponds to the Prokrovski-Talapov line of Fig. 1. Up to now we have found several choices for the constants $\alpha_{A B}$ and $\alpha_{A A}$ for which $H_{0}$ is integrable. We failed to find others, although two other classes of integrable models, namely the chiral Potts 40 and the one with $U_{q} S U(2), q^{3}=1$ symmetry where periodic or semiperiodic representations 41] are taken, have the required $Z_{3}$ symmetry.

Much work is still to be done on the phase structure of the three-state models. One of the questions to be asked is the connection between the case Eq. (6.15), where $\alpha_{A A}=0$, which is known to be massless, to the case $\alpha_{A A}=1$, which is massless as well.

Finally, we mention that in Appendix C (see Eq. (C.16)) we give an example of a $Z_{2}$ symmetric three-state chain (only $A+A \rightarrow \emptyset$ reaction) which is also related to a Hecke algebra. 


\section{Conditions on the reaction rates for the exis- tence of steady states}

Steady states are time-independent solutions of the master equation. In Section 2 we have seen that if the reaction rates satisfy the condition Eq. (2.12), then the empty lattice Eq. (2.13) is a steady state. We now ask for the conditions on the rates such that the probability distribution

$$
\mathcal{P}(\{\beta\})=\prod_{k=1}^{L} f\left(\beta_{k}\right)
$$

is a steady state, where $f(\beta)$ is a given arbitrary function. Our motivation is not only to understand better the structure of the ground states of the master equation and of the corresponding quantum Hamiltonians, but also to be able to address the question of the nonequilibrium behaviour of classical one-dimensional systems. This amounts to introduce a detailed balance condition for a prescribed equilibrium condition $\mathcal{P}$, while in the previous sections we have always taken the empty lattice as the equilibrium configuration. Let us explain this point.

Suppose that at temperature $T$ we have a one-dimensional classical system defined by the probability function

$$
\mathcal{P}(\{\beta\})=\prod_{k=1}^{L} g\left(\alpha_{k}-\alpha_{k+1}\right)
$$

If the $\beta$ 's are site variables, the $\alpha$ 's are link variables.

In our study we are going to consider the two-state model (Ising model) where

$$
g_{2}\left(\beta_{k}\right)=g_{2}\left(\alpha_{k}-\alpha_{k+1}\right)=\exp \left((-1)^{\beta_{k}} / T\right)
$$

and the three-state model (chiral Potts model [42]) where

$$
g_{3}\left(\beta_{k}\right)=g_{3}\left(\alpha_{k}-\alpha_{k+1}\right)=\exp \left(\cos \left(\frac{2 \pi}{3} \beta_{k}+\phi\right) / T\right)
$$

and $\phi$ is a fixed phase. We note the identities

$$
g_{2}(0) g_{2}(1)=1 \quad, \quad g_{3}(0) g_{3}(1) g_{3}(2)=1
$$

If the steady state is given by Eq. (7.2), the master equation describes the evolution of the one-dimensional classical chain from $P(\{\beta\} ; t=0)=P_{0}(\{\beta\})$ to the equilibrium probability distribution $P(\{\beta\} ; t=\infty)=\mathcal{P}(\{\beta\})$.

In order to find the conditions on the states, we first make a similarity transformation Eq. (2.7), taking

$$
\begin{aligned}
h^{(k)}(\alpha) & =f^{1 / 2}(\alpha) \\
W_{\ell, m}(\alpha, \beta) & =\sqrt{\frac{f(\alpha+\ell) f(\beta+m)}{f(\alpha) f(\beta)}} w_{\ell, m}(\alpha, \beta)
\end{aligned}
$$


Then the condition to have Eq (7.1) reads

$$
w_{0,0}(\alpha, \beta)=\sum_{\ell, m=0}^{N-1} w_{\ell, m}(\alpha, \beta) \frac{f(\alpha+\ell) f(\beta+m)}{f(\alpha) f(\beta)}
$$

where the prime indicates that the case $\ell=m=0$ should be excluded. Taking into account the probability conservation condition Eq. (2.4) and making the change of functions

$$
w_{\ell, m}(\alpha, \beta)=\frac{\gamma_{\ell, m}(\alpha, \beta)}{f(\alpha+\ell) f(\beta+m)}
$$

we get the system of equations

$$
\sum_{\ell, m=0}^{N-1}\left(\gamma_{\ell, m}(\alpha, \beta)-\gamma_{\ell, m}(\alpha-\ell, \beta-m)\right)=0
$$

We note that in Eq. (7.9) there is no longer any explicit reference to the function $f$. The whole $f$-dependence is in Eq. (7.8).

We now write down the independent conditions. To do so, it is convenient to introduce the following symmetric and antisymmetric combinations

$$
\begin{aligned}
\gamma_{\ell, m}^{s}(\alpha, \beta) & =\gamma_{\ell, m}(\alpha, \beta)+\gamma_{m, \ell}(\beta, \alpha) \\
\gamma_{\ell, m}^{a}(\alpha, \beta) & =\gamma_{\ell, m}(\alpha, \beta)-\gamma_{m, \ell}(\beta, \alpha)
\end{aligned}
$$

Now, for the two-state system the independent conditions coming from Eq. (7.9) are

$$
\begin{gathered}
\gamma_{1,0}^{s}(0,0)-\gamma_{1,0}^{s}(1,0)=\gamma_{1,0}^{s}(0,1)-\gamma_{1,0}^{s}(1,1)=\gamma_{1,1}(1,1)-\gamma_{1,1}(0,0) \\
\gamma_{1,0}^{a}(1,0)+\gamma_{1,0}^{a}(1,1)+\gamma_{0,1}^{a}(1,0)+\gamma_{0,1}^{a}(0,0)=2 \gamma_{1,1}^{a}(0,1)
\end{gathered}
$$

Note that Eq. (7.11), which relates only symmetric combinations, does not, as expected, contain the diffusion constant since it fixes the time scale only. The antisymmetric diffusion combinations appear however in Eq. (7.12) and thus play a dynamical role. We remind the reader that this property was already noticed in earlier sections when discussing Hamiltonians with quantum group symmetries $(q \neq 1)$.

For the three-state system with $Z_{3}$ symmetry (which means that only the functions $w_{1,2}$ and $w_{2,1}$ appear) we have from Eq. (7.9) the following conditions

$$
\begin{aligned}
\gamma_{2,1}^{s}(0,0)=\gamma_{2,1}^{s}(1,2), \gamma_{2,1}^{s}(1,1) & =\gamma_{2,1}^{s}(2,0), \gamma_{2,1}^{s}(2,2)=\gamma_{2,1}^{s}(0,1) \\
2 \gamma_{2,1}^{a}(1,0) & =\gamma_{2,1}^{a}(2,2)+\gamma_{2,1}^{a}(0,1) \\
2 \gamma_{2,1}^{a}(0,2) & =\gamma_{2,1}^{a}(1,1)+\gamma_{2,1}^{a}(2,0) \\
2 \gamma_{2,1}^{a}(2,1) & =\gamma_{2,1}^{a}(0,0)+\gamma_{2,1}^{a}(1,2)
\end{aligned}
$$

We observe that the detailed balance equations do not determine all the rates. The remaining freedom can be used to fit the experimental data. We shall apply these 
conditions on the rates in the next two sections. Before doing so, we briefly recall the questions of interest and some definitions relevant to critical dynamics. As already mentioned in the introduction, a classical Ising spin system has no intrinsic dynamics, because the Poisson brackets between the spin variables and the Hamiltonian vanish. In a real magnetic system, the spins are interacting with other degrees of freedom (phonons, impurities, ...) and those interactions are responsible for the dynamics of the spins. It is very difficult to implement fully the microscopic dynamics. Accordingly, one replaces the true dynamics by a fictious one given by a master equation for the probability that one spin configuration of the system is realized at a given time. The transition probabilities appearing in the master equation can in principle be computed from the full microscopic dynamics (via projective techniques). Unfortunately, it is often not possible to perform this program, even for simple models [43, 44]. Thus, the transition probabilities are chosen phenomenologically according to two criteria: i) to satisfy detailed balance, necessary to ensure that the desired equilibrium state is stationary, ii) to be qualitatively in agreement with the physics of the system. Once the dynamics is defined, the main problem is to find how the physical quantities will relax towards equilibrium. A quantity of particular interest is the order parameter (the magnetization for the Ising model). Typically, the order parameter relaxes very slowly in the vicinity of a second-order phase transition (critical slowing-down). The dynamical scaling hypothesis assumes that the characteristic time $\tau$ diverges as a power law of the spatial correlation length $\xi ; \tau \sim \xi^{z}$, where $z$ is called the dynamical exponent.

The quantum chain formalism developed here aims to answer two types of questions:

1. Does the dynamical scaling hypothesis $\tau \sim \xi^{z}$ always hold ?

2. What is the status of the "universality" for the dynamical exponent $z$ ?

For one-dimensional systems, the critical point is at zero temperature, $T_{c}=0$. For small $T$, the inverse spatial correlation lengths are

$$
\begin{aligned}
& \xi^{-1}=2 e^{-2 / T}, \quad \text { two-state system } \\
& \xi^{-1}=\frac{3}{2}\left(\frac{g_{3}(1)}{g_{3}(0)}+\frac{g_{3}(2)}{g_{3}(0)}\right), \text { three-state system }
\end{aligned}
$$

For the three-state system we shall only consider the ordinary Potts model, where $\phi=0$ and the so-called superintegrable model 45, where $\phi=\pi / 6$. This serves to illustrate how the dynamics depends on the equilibrium system. In the sequel, the deviation from the critical point will be parametrized in terms of the following masses $\mu \sim \xi^{-1}$, which are related to the temperature as follows

$$
\begin{cases}\mu=e^{-2 / T} & \text { Ising model } \\ \mu=e^{-3 /(2 T)} & \text { Potts model, } \phi=0 \\ \mu=e^{-\sqrt{3} /(2 T)} & \text { superintegrable model, } \phi=\pi / 6\end{cases}
$$


for the three equilibrium models under consideration. In the next two sections we

shall concentrate on the behaviour for small values of $\mu$ in the master equation and in the corresponding Hamiltonians.

\section{Critical dynamics for the Ising model}

We will write the Hamiltonian corresponding to the equilibrium distribution Eq. (7.3) and the conditions Eqs. (7.11, 7.12) for the rates. We assume that the left-right antisymmetric combinations are zero such that Eq. (7.12) is trivially satisfied. To simplify the problem further, we choose the following solution of Eq. (7.11)

$$
\gamma_{1,0}^{s}(0,0)-\gamma_{1,0}^{s}(1,0)=\gamma_{1,0}^{s}(0,1)-\gamma_{1,0}^{s}(1,1)=\gamma_{1,1}(1,1)-\gamma_{1,1}(0,0)=0
$$

With this choice we get

$$
w_{1,0}(1,0)=\mu w_{1,0}(0,0) \quad, \quad w_{1,0}(1,1)=\mu w_{1,0}(0,1) \quad, \quad w_{1,1}(1,1)=\mu^{2} w_{1,1}(0,0)
$$

This tell us that at $T=0(\mu=0)$ the master equation describing the critical dynamics of the Ising model reduces to the master equation considered in Sec. 1 in which annihilation, coagulation and death processes were considered. The particular case with only annihilation was already noticed by Family aand Amar 32].

Taking take $w_{1,1}(1,0)=1$ and by using probability conservation we get

$$
\begin{aligned}
& w_{0,0}(0,0)=\mu^{2} w_{1,1}(0,0)+2 \mu w_{1,0}(0,0) \\
& w_{0,0}(1,0)=1+w_{1,0}(0,0)+\mu w_{1,0}(0,1) \\
& w_{0,0}(1,1)=2 w_{1,0}(0,1)+w_{1,1}(0,0)
\end{aligned}
$$

Using Eqs. (4.47), (4.48) and (8.2) we get the Hamiltonian:

$$
\begin{aligned}
H= & -\frac{1}{2} \sum_{i=1}^{L-1}\left[\sigma_{i}^{x} \sigma_{i+1}^{x}+\sigma_{i}^{y} \sigma_{i+1}^{y}+\Delta \sigma_{i}^{z} \sigma_{i+1}^{z}+\left(1-\Delta^{\prime}\right)\left(\sigma_{i}^{z}+\sigma_{i+1}^{z}\right)\right. \\
& +\mu w_{1,1}(0,0)\left(\sigma_{i}^{x} \sigma_{i+1}^{x}-\sigma_{i}^{y} \sigma_{i+1}^{y}\right) \\
& +2 \mu^{1 / 2} w_{1,0}(0,0)\left(\sigma_{i}^{x} E_{i+1}^{00}+E_{i}^{00} \sigma_{i+1}^{x}\right) \\
& \left.+2 \mu^{1 / 2} w_{1,0}(0,1)\left(\sigma_{i}^{x} E_{i+1}^{11}+E_{i}^{11} \sigma_{i+1}^{x}\right)\right]
\end{aligned}
$$

where

$$
\begin{aligned}
\Delta & =1-\frac{1+\mu^{2}}{2} w_{1,1}(0,0)-(1-\mu) w_{1,0}(0,1)+(1-\mu) w_{1,0}(0,0) \\
\Delta^{\prime} & =\Delta-\mu w_{1,0}(0,1)+\mu^{2} w_{1,1}(0,0)-(1-2 \mu) w_{1,0}(0,0)
\end{aligned}
$$

This result is very interesting and deserves a few comments. If $w_{1,0}(0,0) \neq 0$, even for $\mu=0$ (when the equilibrium system is at the critical temperature $T=0$ ) we 
still have $\Delta^{\prime}<\Delta$ and consequently the time-dependent system is massive with an exponential fall-off in the correlation functions. This is to the best of our knowledge the first example of a system having an equilibrium second order phase transition but no critical slowing down. It is not obvious that this phenomenon generalizes into higher dimensions since only in one dimension, at $T=T_{c}$ the system is fully ordered.

On the other hand if $w_{1,0}(0,0)=0$, but $w_{1,0}(0,1)$ is non-zero, we would expect the relaxation time should be

$$
\tau^{-1} \sim \mu
$$

The reason is simple and can be understood using perturbative arguments. We get terms of order $\mathcal{O}(\mu)$ in $\Delta^{\prime}$, which couples to the $U(1)$ scalars, $\sigma_{i}^{z}+\sigma_{i+1}^{z}$, in the Hamiltonian. The terms present in the Hamiltonian which are of order $\mathcal{O}\left(\mu^{1 / 2}\right)$ occur in combinations like $\mu^{1 / 2} w_{1,0}(0,1)$ which do not couple to $U(1)$ scalars and should thus only contribute in second order. The contribution of order $\mathcal{O}(\mu)$ can be only eliminated when a $Z_{2}$ symmetry is present in the problem. This implies that

$$
w_{1,0}(0,0)=w_{1,0}(0,1)=0
$$

and by repeating the same argument we find

$$
\tau^{-1} \sim \mu^{2}
$$

However these perturbative arguments should be considered with some care since they apply to chains of a given length L. It might happen that in the large L limit, the leading term in the power expansions in $\mu$ has vanishing coefficients and we have to consider the next leading order term. To be more explicit, in a concrete calculation of the case $w_{1,0}(0,1) \neq 0, w_{1,0}(0,0)=w_{1,1}(0,0)=0$ for a given chain one obtains:

$$
\tau_{L}^{-1}=a_{L}+b_{L} \mu+c_{L} \mu^{2}+\ldots
$$

In the large $\mathrm{L}$ limit $a_{L}$ must vanish (we end up in a massless system at $\mu=0$ ) but the same can also happen to $b_{L}$. It turns out that this is indeed happening since we can derive the exact result using the calculations of Ref. 30] for the coagulationdecoagulation chemical process and find

$$
\tau^{-1}=\frac{\mu^{2}}{4}
$$

We would like to emphasize that the calculation of the coefficients $a_{L}, b_{L}, c_{L}, \ldots$ can be difficult in general so that numerical estimates might be useful here. For the case $w_{1,1}(0,0) \neq 0$ and $w_{1,0}(0,1)=w_{1,0}(0,0)$ the perturbative argument is correct and Eq. 8.8) stays valid as we can see from the exact result of Ref. 31. We conclude that in the Ising case the critical exponent $z=2$ is universal. 


\section{Three-states critical dynamics}

Turning back to the three-states models, we now assume, in analogy to the two-state case, that the assymmetric rates in Eq. (7.14) are zero and we thus only have to solve Eq. (7.13). We begin by considering the ordinary Potts model, with $\phi=0$. We find

$$
w_{2,1}(1,2)=\mu^{2} w_{2,1}(0,0), w_{2,1}(1,1)=\mu w_{2,1}(2,0) \quad, \quad w_{2,1}(2,2)=\mu w_{2,1}(0,1)
$$

This leads to the following Hamiltonian

$$
\begin{aligned}
H= & \sum_{i=1}^{L-1}\left[2 \mu^{2} w_{2,1}(0,0) E_{i}^{00} E_{i+1}^{00}+2 w_{2,1}(2,0) E_{i}^{11} E_{i+1}^{11}+2 w_{2,1}(0,1) E_{i}^{22} E_{i+1}^{22}\right. \\
& +\left(w_{2,1}(1,0)+\mu w_{2,1}(0,1)\right)\left(E_{i}^{00} E_{i+1}^{11}+E_{i}^{11} E_{i+1}^{00}\right) \\
& +\left(w_{2,1}(0,2)+\mu w_{2,1}(2,0)\right)\left(E_{i}^{00} E_{i+1}^{22}+E_{i}^{22} E_{i+1}^{00}\right) \\
& +\left(w_{2,1}(2,1)+w_{2,1}(0,0)\right)\left(E_{i}^{11} E_{i+1}^{22}+E_{i}^{22} E_{i+1}^{11}\right) \\
& -\mu w_{2,1}(0,0)\left(E_{i}^{10} E_{i+1}^{20}+E_{i}^{01} E_{i+1}^{02}+E_{i}^{20} E_{i+1}^{10}+E_{i}^{02} E_{i+1}^{01}\right) \\
& -\mu^{1 / 2} w_{2,1}(2,0)\left(E_{i}^{10} E_{i+1}^{12}+E_{i}^{01} E_{i+1}^{21}+E_{i}^{21} E_{i+1}^{01}+E_{i}^{12} E_{i+1}^{10}\right) \\
& -\mu^{1 / 2} w_{2,1}(0,1)\left(E_{i}^{02} E_{i+1}^{12}+E_{i}^{20} E_{i+1}^{21}+E_{i}^{21} E_{i+1}^{20}+E_{i}^{12} E_{i+1}^{02}\right) \\
& -w_{2,1}(1,0)\left(E_{i}^{10} E_{i+1}^{01}+E_{i}^{01} E_{i+1}^{10}\right) \\
& -w_{2,1}(2,1)\left(E_{i}^{21} E_{i+1}^{12}+E_{i}^{12} E_{i+1}^{21}\right) \\
& \left.-w_{2,1}(0,2)\left(E_{i}^{02} E_{i+1}^{20}+E_{i}^{20} E_{i+1}^{02}\right)\right]
\end{aligned}
$$

In writing Eq. (9.2), we have used the similarity transformation $(2.8)$ with $h^{(k)}(1) / h^{(k)}(0)=$ $h^{(k)}(2) / h^{(k)}(0)=\sqrt{\mu}$. We are thus left, after choosing the time scale, with five free parameters. If we now take $\mu=0$ in Eq. (9.2), we get back to the chains studied in Section 6. If we make the choice of coupling constants from Eqs. (6.10, 6.12), at $\mu=0$ we find that $H$ coincides with the $H_{0}$ of Eq. (6.13). We can thus write

$$
H=H_{0}+\tilde{H}_{1}
$$

where $\tilde{H}_{1}$ is in the case $\alpha_{A A}=\alpha_{B B}$

$$
\begin{aligned}
\tilde{H}_{1}= & \sum_{i=1}^{L-1}\left[2 \mu^{2} \alpha_{A B} E_{i}^{00} E_{i+1}^{00}+\mu \alpha_{A A}\left(\left(\mathbf{1}-\rho^{0}\right)_{i} \rho_{i+1}^{0}+\rho_{i}^{0}\left(\mathbf{1}-\rho^{0}\right)_{i+1}\right)\right. \\
& -\mu \alpha_{A B}\left(E_{i}^{10} E_{i+1}^{20}+E_{i}^{01} E_{i+1}^{02}+E_{i}^{20} E_{i+1}^{10}+E_{i}^{02} E_{i+1}^{01}\right) \\
& -\mu^{1 / 2} \alpha_{A A}\left(\left(E^{10}+E^{02}\right)_{i} E_{i+1}^{12}+E_{i}^{12}\left(E^{10}+E^{02}\right)_{i+1}\right. \\
& \left.\left.+\left(E^{01}+E^{20}\right)_{i} E_{i+1}^{21}+E_{i}^{21}\left(E^{01}+E^{20}\right)_{i+1}\right)\right]
\end{aligned}
$$


and

$$
\rho^{0}=\left(\begin{array}{lll}
1 & 0 & 0 \\
0 & 0 & 0 \\
0 & 0 & 1
\end{array}\right)=E^{00}+E^{22}
$$

Notice that the terms which couple to $\mu \alpha_{A A}$ keep the $U(1) \otimes U(1)$ symmetry of the unperturbed problem. If this is the only perturbation present, we should thus get

$$
\tau^{-1} \sim \mu
$$

This result is similar to the behaviour observed for the Ising model (see Eq. (8.6)). We would like to stress that this result might be "naive" (see the discussion after Eq. (8.8) in Sec. 8).

We now consider the superintegrable model, where $\phi=\pi / 6$. This case is interesting because $g_{3}(2)=1$ and this will have important consequences. From Eq. (7.13), we find

$$
w_{2,1}(1,2)=\mu^{3} w_{2,1}(0,0), w_{2,1}(1,1)=\mu^{3} w_{2,1}(2,0) \quad, \quad w_{2,1}(2,2)=w_{2,1}(0,1)
$$

The Hamiltonian is

$$
\begin{aligned}
H= & \sum_{i=1}^{L-1}\left[2 \mu^{3} w_{2,1}(0,0) E_{i}^{00} E_{i+1}^{00}+2 w_{2,1}(2,0) E_{i}^{11} E_{i+1}^{11}+2 w_{2,1}(0,1) E_{i}^{22} E_{i+1}^{22}\right. \\
& +\left(w_{2,1}(1,0)+w_{2,1}(0,1)\right)\left(E_{i}^{00} E_{i+1}^{11}+E_{i}^{11} E_{i+1}^{00}\right) \\
& +\left(w_{2,1}(0,2)+\mu^{3} w_{2,1}(2,0)\right)\left(E_{i}^{00} E_{i+1}^{22}+E_{i}^{22} E_{i+1}^{00}\right) \\
& +\left(w_{2,1}(2,1)+w_{2,1}(0,0)\right)\left(E_{i}^{11} E_{i+1}^{22}+E_{i}^{22} E_{i+1}^{11}\right) \\
& -\mu^{3 / 2} w_{2,1}(0,0)\left(E_{i}^{10} E_{i+1}^{20}+E_{i}^{20} E_{i+1}^{10}+E_{i}^{01} E_{i+1}^{02}+E_{i}^{02} E_{i+1}^{01}\right) \\
& -\mu^{3 / 2} w_{2,1}(2,0)\left(E_{i}^{10} E_{i+1}^{12}+E_{i}^{01} E_{i+1}^{21}+E_{i}^{21} E_{i+1}^{01}+E_{i}^{12} E_{i+1}^{10}\right) \\
& -w_{2,1}(0,1)\left(E_{i}^{02} E_{i+1}^{12}+E_{i}^{20} E_{i+1}^{21}+E_{i}^{21} E_{i+1}^{20}+E_{i}^{12} E_{i+1}^{02}\right) \\
& -w_{2,1}(1,0)\left(E_{i}^{10} E_{i+1}^{01}+E_{i}^{01} E_{i+1}^{10}\right) \\
& -w_{2,1}(2,1)\left(E_{i}^{21} E_{i+1}^{12}+E_{i}^{12} E_{i+1}^{21}\right) \\
& \left.-w_{2,1}(0,2)\left(E_{i}^{02} E_{i+1}^{20}+E_{i}^{20} E_{i+1}^{02}\right)\right]
\end{aligned}
$$

Notice that for $\mu=0$ we got a new term in Eq. (9.8) which did not exist in the Hamiltonians studied in Section 6 and which reads

$$
w_{2,1}(0,1)\left(\rho_{i}^{+} \sigma_{i+1}^{+}+\rho_{i}^{-} \sigma_{i+1}^{-}+\sigma_{i}^{-} \rho_{i+1}^{-}+\sigma_{i}^{+} \rho_{i+1}^{+}\right)
$$

where we have used the notation from Eq. (6.20). It is not known whether the Hamiltonian Eq. (9.8) for $\mu=0$ is critical or not. Comparing the ordinary Potts and the superintegrable Potts cases we notice that the number of independent rates 
is different. We also see, by comparing Eqs. (9.2) with (9.9) that $\mu^{1 / 2}$ is replaced by $\mu^{3 / 2}$, consequently the dynamical exponents changes. We conclude that when moving from the Potts model (symmetry $S_{3}$ ) to the superintegrable chiral Potts model (symmetry $Z_{3}$ ), the exponents also changes.

We have seen how the universal critical exponents can be predicted from considering the structure of integrable Hamiltonians at $\mu=0$ and appealling to perturbation theory. Here again, we have implicitly assumed that all the six rates $w_{2,1}(0,0), w_{2,1}(2,0), w_{2,1}(0,1), w_{2,1}(1,0), w_{2,1}(2,1)$ and $w_{2,1}(0,2)$ have finite, non-vanishing limits as $\mu \rightarrow 0$. Otherwise, one may create any value of some effective exponent like in Refs. 18, 43, 44.

\section{Conclusions}

We have started our study asking ourselves what we thought is a simple question: is the present progress achieved in the understanding of one-dimensional integrable quantum chains useful for solving master equations describing the dynamics of classical one-dimensional spin systems ? We find plenty of evidence for a positive answer. At same time, after finishing this long paper, we have the feeling that we are just at the beginning of a long path.

Although initially we thought that our task would be just to use the available mathematical knowledge of integrable systems to find physical results, the physical problems brought a lot of feed-back into mathematics. Studying open chains with particular transitions rates lead us, to our surprise, automatically to new, hermitian and non-hermitian, representations of interesting associative algebras. We remind the reader that in equlibrium problems, quantum groups and associative algebras appear only through rather artificial boundary conditions. The physical applications of non-hermitian representations of associative algebras appear for the first time in our context. More about this subject will be published elsewhere.

We would like to stress once more that although the phase diagram in the space of transition rates can be easily obtained from the Hamiltonian spectrum computed for example by using the Bethe ansatz, the calculation of nonequilibrium averages (which are not those normally occuring in equilibrium statistical physics) might pose formidable problems.

For the physical understanding of reaction-diffusion processes or of simple critical dynamics we think that we went beyond the particular cases studied up to now in the literature.

As the reader has certainly noticed while going through Sections 1 and 6, there is plenty of room for more work especially for three-states models, where we have only considered systems with $Z_{3}$ symmetry and even in this case, the whole phase diagram is not yet completely known.

Our experience with the study of properties of quantum chains for equlibrium purposes lead us to repeat analogous questions for nonequlibrium problems. For ex- 
ample, in the simple model studied in Section 5 we have verified that finite-size scaling applies also to nonequlibrium situations. This opens the possibility of using standard numerical methods of matrix diagonalization of finite systems, for computing critical exponents. The solutions found in Section 7 for the detailed-balance equations should be useful for other dynamical processes than those discussed in Sections 8 and 9, in a better understanding of critical dynamics.

\section{Acknowledgements}

We would like to thank C. Gómez, V. Privman and G. Schütz for useful discussions. We are most grateful to Klaus Krebs, Markus Pfannmülller and Birgit Wehefritz for the excellent job they did critically reading the manuscript. F.A. thanks the Fundação de Amparo à Pesquisa do Estado de São Paulo - FAPESP - Brasil and the Deutsche Forschungsgemeinschaft - DFG - Germany for support. M.D. and M.H. thank the Swiss National Science Foundation for support. V.R. would like to thank CERN, the University of Geneva, the Einstein Center of the Weizmann Institute and SISSA for the warm hospitality he has enjoyed and has enabled him to participate in this project.

\section{Appendix A. Eigenvectors of some special non-hermitian Hamiltonians}

We discuss the treatment of a certain class of non-hermitian Hamiltonians with the structure

$$
H=H_{0}+H_{1}
$$

where $H_{0}$ is hermitian and has the same spectrum as $H$. Moreover we are interested in the case where the eigenspectrum is non-degenerated. Suppose the eigenvectors $\left|u_{i}\right\rangle$ and the eigenvalues $\lambda_{i}$ of $H_{0}$

$$
H_{0}\left|u_{i}\right\rangle=\lambda_{i}\left|u_{i}\right\rangle
$$

are known and we want to find the eigenvectors of $H$. This problem is of interest for both analytical and numerical (finite-size scaling) calculations in reaction-diffusion processes (see Eqs. (4.11), (4.31) and (6.11)). In the basis $\left\{\left|u_{i}\right\rangle\right\}, H_{1}$ has only the following non-vanishing matrix elements

$$
\left\langle u_{i}\left|H_{1}\right| u_{j}\right\rangle=G_{i j} ; i<j, i, j=1, \ldots, N
$$


Thus the eigenvalues of $H$ are again $\lambda_{1}, \ldots, \lambda_{N}$. Let $\left|v_{i}\right\rangle$ be the eigenvector of $H$ corresponding to the eigenvalue $\lambda_{i}$. It can be written as

$$
\left|v_{i}\right\rangle=\sum_{i \geq j} A_{i j}\left|u_{j}\right\rangle
$$

with $A_{i i}=1$ and the graphical rule to compute the $A_{i, j}(i>j)$ is obvious if we give the first few

$$
\begin{aligned}
A_{21}= & \frac{G_{12}}{\lambda_{2}-\lambda_{1}}, A_{32}=\frac{G_{23}}{\lambda_{3}-\lambda_{2}}, A_{31}=\frac{G_{13}}{\lambda_{3}-\lambda_{1}}+\frac{G_{12} G_{23}}{\left(\lambda_{3}-\lambda_{2}\right)\left(\lambda_{3}-\lambda_{1}\right)} \\
A_{43}= & \frac{G_{34}}{\lambda_{4}-\lambda_{3}}, A_{42}=\frac{G_{24}}{\lambda_{4}-\lambda_{2}}+\frac{G_{23} G_{34}}{\left(\lambda_{4}-\lambda_{3}\right)\left(\lambda_{4}-\lambda_{2}\right)} \\
A_{41}= & \frac{G_{14}}{\lambda_{4}-\lambda_{1}}+\frac{G_{13} G_{34}}{\left(\lambda_{4}-\lambda_{3}\right)\left(\lambda_{4}-\lambda_{1}\right)}+\frac{G_{12} G_{24}}{\left(\lambda_{4}-\lambda_{2}\right)\left(\lambda_{4}-\lambda_{1}\right)} \\
& +\frac{G_{12} G_{23} G_{34}}{\left(\lambda_{4}-\lambda_{3}\right)\left(\lambda_{4}-\lambda_{2}\right)\left(\lambda_{4}-\lambda_{1}\right)}
\end{aligned}
$$

\section{Appendix B. The seven-vertex model}

As an example, we shall consider in more detail one of the models related to a Hecke algebra (see Appendix C) in order to obtain the Boltzmann weights of an associated two-dimensional vertex model and thus prove its integrability.

The model we consider is the one introduced in Section 1 which describes diffusion and pairwise annihilation of $A$ particles, see Eq. (4.8). We choose the special tuning

which makes $\Delta=0$ and take the functions $\varphi^{(k)}(\alpha, \beta)=1$ where $\alpha, \beta=0,1$. The Hamiltonian is given by

$$
H=D\left(H_{0}+H_{1}\right)
$$

where

$$
\begin{aligned}
& H_{0}=-\sum_{i=1}^{L-1}\left[q E_{i}^{01} E_{i+1}^{10}+\frac{1}{q} E_{i}^{10} E_{i+1}^{01}+\frac{q}{2}\left(\sigma_{i}^{z}-1\right)+\frac{1}{2 q}\left(\sigma_{i+1}^{z}-1\right)\right] \\
& H_{1}=-\Omega \sum_{i=1}^{L-1} E_{i}^{01} E_{i+1}^{01}
\end{aligned}
$$

and

$$
\Omega=\frac{w_{1,1}(0,0)}{D}, q=\sqrt{\frac{w_{1,1}(1,0)}{w_{1,1}(0,1)}}, \quad D=\sqrt{w_{1,1}(0,1) w_{1,1}(1,0)}
$$

Doing the canonical transformation $E_{i}^{k \ell}=(-1)^{k-\ell} E_{i}^{k \ell}$, only at even sites $i$, this Hamiltonian in the $\sigma^{z}$ basis takes the simple form

$$
H=-D \sum_{i=1}^{L-1} e_{i}
$$


where

$$
e_{i}=\mathbf{1}_{1} \otimes \ldots \otimes \mathbf{1}_{i-1} \otimes\left(\begin{array}{cccc}
0 & 0 & 0 & \Omega \\
0 & \frac{1}{q} & q & 0 \\
0 & \frac{1}{q} & q & 0 \\
0 & 0 & 0 & q+\frac{1}{q}
\end{array}\right) \otimes \mathbf{1}_{i+2} \otimes \ldots
$$

and $i=1,2, \ldots, L-1$ and $\mathbf{1}_{i}$ are $2 \times 2$ unit matrices attached to the site $i$.

The Hamiltonian Eq. (B.4) is known to be integrable through a Jordan-Wigner transformation (see Section 5), we think however that the approach given here gives not only new insight into the problem but is of a larger validity.

We can show that the above matrices $e_{i}, i=1, \ldots, L$ satisfy the Hecke algebra, for arbitrary values of $\Omega$

$$
\begin{aligned}
& e_{i} e_{i \pm 1} e_{i}-e_{i}=e_{i \pm 1} e_{i} e_{i \pm 1}-e_{i \pm 1} \\
& {\left[e_{i}, e_{j}\right]=0 ;|i-j| \geq 2} \\
& e_{i}^{2}=\left(q+q^{-1}\right) e_{i}
\end{aligned}
$$

This is the first example we know of where nonhermitian (take $q$ real) representations of the Hecke algebra appear in physical applications. The hermitian case $\Omega=0$ corresponds to the quantum chain introduced by Saleur [46].

Due to the algebraic properties Eq. (B.6) we can construct an associated twodimensional vertex model having a row-to-row transfer matrix depending on the spectral parameter $\theta$. There transfer matrices will satisfy the Yang-Baxter equations [47] which implies that they commute among themselves for different values of the spectral parameter.

In order to obtain the configuration and the Boltzmann weights associated to this vertex model, we need the spectral parameter dependent matrix $\check{R}_{i}(\theta), i=1,2, \ldots$. This is found by the Baxterization procedure [21] for Hecke algebras, namely

$$
\check{R}_{i}(\theta)=\frac{\sinh \theta}{\sinh \eta} e_{i}+\frac{\sinh (\eta-\theta)}{\sinh \eta}, q=e^{\eta}
$$

which gives us

$\check{R}_{i}(\theta)=\mathbf{1}_{1} \otimes \ldots \otimes \mathbf{1}_{i-1} \otimes \frac{1}{\sinh \eta}\left(\begin{array}{cccc}\sinh (\eta-\theta) & & \Omega \sinh \theta \\ & e^{-\theta} \sinh \eta & e^{\eta} \sinh \theta & \\ & e^{-\eta} \sinh \theta & e^{\theta} \sinh \eta & \\ & & & \sinh (\eta+\theta)\end{array}\right) \otimes \mathbf{1}_{i+2} \otimes \ldots$

The relations Eq. (B.6) imply that these matrices satisfy the spectral parameter dependent braid group relations

$$
\begin{aligned}
\check{R}_{i}(\theta) \check{R}_{i \pm 1}\left(\theta+\theta^{\prime}\right) \check{R}_{i}\left(\theta^{\prime}\right) & =\check{R}_{i \pm 1}\left(\theta^{\prime}\right) \check{R}_{i}\left(\theta+\theta^{\prime}\right) \check{R}_{i \pm 1}(\theta) \\
{\left[\check{R}_{i}(\theta), \check{R}_{j}\left(\theta^{\prime}\right)\right] } & =0,|i-j| \geq 2
\end{aligned}
$$


which are equivalent to the Yang-Baxter equations.

The Boltzmann weights $S_{\ell, m}^{k n}$ of the vertex configuration labelled by $(k, \ell, m, n)$ in the associated vertex model can be obtained from the relation

$$
\check{R}_{i}(\theta)=S_{\ell, m}^{k, n} \mathbf{1}_{1} \otimes \ldots \otimes \mathbf{1}_{i-1} \otimes E^{m k} \otimes E^{n \ell} \otimes \mathbf{1}_{i+2} \otimes \ldots
$$

This implies that the vertex model associated to Eq. (B.1) is a seven vertex model. If we denote by an index zero a down (or left) arrow and by an index one an up (or right) arrow, the vertex configurations with their Boltzmann weights are given in Figure 2. We also show in this figure the corresponding chemical processes related to each vertex. The vertices 1 to 4 correspond to no reaction, vertices 5 and 6 to diffusion to the right and the left and vertex 7 to the pair annihilation process. The derivative of the logarithm of the row-to-row transfer matrix, with these Boltzmann weights and evaluated at $\theta=0$, gives back the Hamiltonian Eq. (B.1).

\section{Appendix C. Hecke algebra and reaction-diffusion processes}

In this appendix we define the Hecke algebra and give some examples of Hamiltonians, related to dynamical processes, which are representations of this algebra.

The Hecke algebra is an associative algebra with generators $e_{i}(i=1, \ldots, L-1)$ satisfying the relations

$$
\begin{aligned}
& e_{i} e_{i \pm 1} e_{i}-e_{i}=e_{i \pm 1} e_{i} e_{i \pm 1}-e_{i \pm 1} \\
& {\left[e_{i}, e_{j}\right]=0 ;|i-j| \geq 2} \\
& e_{i}^{2}=\left(q+q^{-1}\right) e_{i}
\end{aligned}
$$

where $q$ is a complex parameter.

One of the main features of the above algebra is related to the fact that a spectraldependent $\check{R}(u)$ matrix, satisfying the Yang-Baxter relations [47, can be constructed in a standard form. As a consequence of this procedure, also called "Baxterization"

[21], the Hamiltonian $\sum_{i=1}^{L-1} e_{i}$, as well as its associated vertex model, has an infinite number of conservation laws and we expect, in general, its exact integrability. In Appendix B we give an example of this "Baxterization" procedure and derive the associated vertex model for one of the chains considered in this paper.

It is important to stress here that distinct Hamiltonians satisfying the same Hecke algebra may correspond to different representations of the algebra. They will share, apart from degeneracies (which may be zero) the same eigenenergies. These chains will have a massive or massless behaviour depending on the value of $q$. In particular, for $q$ real they will always have a massive behaviour except for $q=1$, where they will be massless.

The $e_{i}$ 's appearing in quantum chains quantum chains satisfy beyond Eqs. (C.1 C.3) additional relations which define quotients of the Hecke algebra. Obviously, 
quantum chains obeying the same quotient have more coincidences in their spectra, see 23] for details. A well-known quotient is the Temperley-Lieb algebra 48] defined by (C.2,C.3) and

$$
e_{i} e_{i \pm 1} e_{i}=e_{i}, \quad e_{i \pm 1} e_{i} e_{i \pm 1}=e_{i \pm 1} \quad ; \quad i=1,2, \ldots
$$

A realization of this algebra is given by the XXZ chain with surface magnetic fields (invariant under the $U_{q} S U(2 / 0)$ quantum group) and the quantum Potts chain with free ends 48, 49]. In Sections 1 and 6 we have used the notation $U_{q} S U(n)$ for $U_{q} S U(n / 0)$. With the new notation $U_{q} S U(n / m)$ with $m \neq 0$ corresponds to quantum superalgebras [50.) Another less known quotient is defined by (C.1-C.3) and the additional relation [23]

$$
\left(e_{i} e_{i+2}\right) e_{i+1}\left(q+q^{-1}-e_{i}\right)\left(q+q^{-1}-e_{i+2}\right)=0 ; i=1,2, \ldots
$$

which has a realization in the two-colors Perk-Schultz model [22], invariant under the quantum group $U_{q} S U(1 / 1)$.

In this paper we show that several quantum chains, related to chemical processes, are realizations of the Hecke algebra (C.1)-(C.3). As a general outcome from our analysis we verifyed that as long as only diffusion and interchange of particle processes (the number of particles in each species is conserved separately) are allowed, these realizations arise quite naturally. In the cases where other processes are also allowed the chain in general will not satisfy the Hecke algebra. However, as we shall see, for certain processes and special tunings of transition rates, the corresponding chains will turn out to satisfy the Hecke algebra again.

Although we can generalize our results to an arbitrary number of different chemical species, following the line of this paper we will consider here only the cases where we have, beyond vacancies, 1 species $(A)$ or 2 species $(A$ and $B)$.

Let us consider initially the cases where we have only particles and vacancies, see Section 4 . If we only allow the diffusion process with the transition rates satisfying Eqs. (4.9), (4.11) we obtain the XXZ chain with anisotropy $\Delta=\left(q+q^{-1}\right) / 2$

$$
H / D=H_{0}=\sum_{i=1}^{L} e_{i}
$$

where

$$
\begin{aligned}
e_{i}= & -\frac{1}{2}\left[\sigma_{i}^{x} \sigma_{i+1}^{x}+\sigma_{i}^{y} \sigma_{i+1}^{y}+\frac{1}{2}\left(q+\frac{1}{q}\right) \sigma_{i}^{z} \sigma_{i+1}^{z}+\frac{1}{2}\left(q-\frac{1}{q}\right)\left(\sigma_{i}^{z}-\sigma_{i+1}^{z}\right)\right. \\
& \left.-\frac{1}{2}\left(q+\frac{1}{q}\right)\right] ; i=1, \ldots, L-1
\end{aligned}
$$

We can verify that these $e_{i}$ matrices are just a $2^{L}$-dimensional representation of the Hecke algebra. Moreover, this Hamiltonian satisfies the quotient defining the 
Temperley-Lieb algebra (C.4). It is important to stress that the terms proportional to $\sigma_{i}^{z}$ appear naturally in (C.7) due to the diffusion mechanisms. These terms, although not present in a periodic chain, are crucial in order to generate the Temperley-Lieb algebra. The particular chain (C.7) is invariant under the quantum group $U_{q} S U(2 / 0)$.

Another example, also with only particles and vacancies, appears when beyond diffusion we also allow the annihilation process $A+A \rightarrow \emptyset+\emptyset$ (with rate $w_{1,1}(0,0)$ ). In this case, by making the special tuning

$$
w_{1,1}(0,0)=w_{1,1}(0,1)+w_{1,1}(1,0)
$$

and choosing the functions $\varphi^{(k)}(\ell, m)=1$ we obtain

$$
H / D=\sum_{i=1}^{L-1} e_{i}
$$

where

$$
\begin{aligned}
e_{i}= & -q E_{i}^{01} E_{i+1}^{10}-\frac{1}{q} E_{i}^{10} E_{i+1}^{01}-\left(q+\frac{1}{q}\right) E_{i}^{01} E_{i+1}^{01} \\
& +\frac{q}{2}\left(\sigma_{i}^{z}-1\right)+\frac{1}{2 q}\left(\sigma_{i+1}^{z}-1\right) ; i=1, \ldots, L-1
\end{aligned}
$$

and

$$
q=\sqrt{\frac{w_{1,1}(1,0)}{w_{1,1}(0,1)}}, \quad D=\sqrt{w_{1,1}(0,1) w_{1,1}(1,0)}
$$

These matrices $e_{i}$ satisfy the Hecke relations (C.1)-(C.3) and are also generators of the quotient defined by Eq. (C.5). This is the first example we are aware of in which nonhermitian ( $q$ real) representations of the Hecke algebra appear in a physical context. In Appendix B we derive the vertex model associated to this chain.

Let us now consider some cases where we have two types of particles ( $A$ and $B$ ). If we consider only the process of diffusion and interchange of particles, as we saw in Section 6, by choosing the diffusion rates Eq. (6.4) we obtain the anisotropic Sutherland model [36]

$$
H / D=\sum_{i=1}^{L-1} e_{i}
$$

where

$$
\begin{aligned}
e_{i}= & \frac{1}{2}\left(q+\frac{1}{q}\right)-\left[\sum_{a, b=0 ; a \neq b}^{2} E_{i}^{a b} E_{i+1}^{b a}+\frac{1}{2}\left(q+\frac{1}{q}\right) \sum_{a=0}^{2} E_{i}^{a a} E_{i+1}^{a a}\right. \\
& \left.+\frac{1}{2}\left(q-\frac{1}{q}\right) \sum_{a, b=0 ; a \neq b}^{2} \operatorname{sign}(a-b) E_{i}^{a a} E_{i+1}^{b b}\right]
\end{aligned}
$$

which again satisfy the Hecke algebra. The above Hamiltonian is invariant under the quantum group $U_{q} S U(3 / 0)$ (we do not give the corresponding quotient here). 
Let us now consider the case where beyond the above processes we also include the annihilation $A+A \rightarrow \emptyset+\emptyset$ (rate $w_{1,1}(0,0)$ ). If we use the relation (6.5) between the diffusion rates and the condition

$$
w_{1,1}(0,0)=\Gamma_{L}+\Gamma_{R}
$$

we obtain

$$
H / D^{\prime}=\sum_{i=1}^{L-1} e_{i}
$$

where

$$
\begin{aligned}
e_{i}= & -\left[\frac{1}{q} E_{i}^{01} E_{i+1}^{10}+q E_{i}^{10} E_{i+1}^{01}+\frac{1}{q} E_{i}^{02} E_{i+1}^{20}\right. \\
& \left.+q E_{i}^{20} E_{i+1}^{02}+\frac{1}{q} E_{i}^{12} E_{i+1}^{21}+q E_{i}^{21} E_{i+1}^{12}+\Omega E_{i}^{01} E_{i+1}^{01}\right] \\
& +q\left(E_{i}^{00} E_{i+1}^{11}+E_{i}^{00} E_{i+1}^{22}+E_{i}^{11} E_{i+1}^{22}\right)+\frac{1}{q}\left(E_{i}^{11} E_{i+1}^{00}+E_{i}^{22} E_{i+1}^{00}+E_{i}^{22} E_{i+1}^{11}\right) \\
& +\left(q+\frac{1}{q}\right) E_{i}^{11} E_{i+1}^{11}
\end{aligned}
$$

and

$$
\Omega=q+\frac{1}{q}, q=\sqrt{\Gamma_{R} / \Gamma_{L}}, D^{\prime}=\sqrt{\Gamma_{R} \Gamma_{L}}
$$

The (nonhermitian!) operators $e_{i}$ also satisfy the Hecke algebra. In fact we verified that this is a property of (C.16)-(C.17) for arbitrary values of $\Omega$. For $\Omega=0$ the Hamiltonian reduces to the three-color Perk-Schultz model 22] (which has the quantum superalgebra $U_{q} S U(2 / 1)$ as symmetry) and it is also related to a special point of the $t-J$ model [51] where exact integrability takes place [52].

Let us return to the case where we do not have annihilation, see Eqs. (C.9,C.11). If we now forbid the process where the particles interchange positions $(A+B \leftrightarrow B+A)$

$$
w_{1,2}(1,2)=w_{2,1}(2,1)=0
$$

we obtain

$$
H / D^{\prime}=\sum_{i=1}^{L-1} e_{i}
$$

with

$$
\begin{aligned}
e_{i}= & -\left[\sum_{a=1}^{2}\left(E_{i}^{0 a} E_{i+1}^{a 0}+E_{i}^{a 0} E_{i+1}^{0 a}\right)+q E i^{00}\left(E_{i+1}^{11}+E_{i+1}^{22}\right)\right. \\
& \left.-\frac{1}{q}\left(E_{i}^{11}+E_{i}^{22}\right) E_{i+1}^{00}\right] ; i=1, \ldots, L-1
\end{aligned}
$$


This model was introduced in [53] and the generators satisfy the Hecke algebra. Moreover we verified that like the $U_{q} S U(2 / 0)$ model (C.6.C.7) the matrices $e_{i}$ are also the generators of a $3^{L}$-dimensional Temperley-Lieb algebra. The symmetry of the chain (C.20) is known and described in Ref. [55].

If we now include (see Eq. 6.11)) the annihilation processes $A+B \rightarrow \emptyset+\emptyset$, $A+A \rightarrow \emptyset+B, B+B \rightarrow \emptyset+A$ with the reaction rates related in the special way

$$
\begin{gathered}
w_{2,1}(1,0)=w_{1,2}(2,0)=w_{2,1}(2,0)=w_{1,2}(1,0)=\Gamma_{L} \\
w_{1,2}(0,1)=w_{2,1}(0,2)=w_{1,2}(0,2)=w_{2,1}(0,1)=\Gamma_{R} \\
w_{1,2}(0,0)=w_{2,1}(0,0)=\Gamma_{R}+\Gamma_{L} \\
q=\sqrt{\Gamma_{L} / \Gamma_{R}}, D^{\prime}=\sqrt{\Gamma_{L} \Gamma_{R}}
\end{gathered}
$$

we obtain

$$
H / D^{\prime}=H_{0} / D^{\prime}+H_{1} / D^{\prime}=\sum_{i=1}^{L-1} e_{i}, \quad e_{i}=e_{i}^{0}+e_{i}^{1}
$$

where

$$
\begin{aligned}
e_{i}^{0}= & \sum_{a=1}^{2}\left(q E_{i}^{00} E_{i+1}^{a a}+\frac{1}{q} E_{i}^{a a} E_{i+1}^{00}-q E_{i}^{a 0} E_{i+1}^{0 a}-\frac{1}{q} E_{i}^{0 a} E_{i+1}^{a 0}\right) \\
& +\left(q+\frac{1}{q}\right) \sum_{a, b=1}^{2} E_{i}^{a a} E_{i+1}^{b b} ; i=1, \ldots, L-1
\end{aligned}
$$

and

$$
\begin{aligned}
e_{i}^{1}= & -\left(\frac{1}{q} E_{i}^{01} E_{i+1}^{21}+q E_{i}^{21} E_{i+1}^{01}+q E_{i}^{12} E_{i+1}^{02}+\frac{1}{q} E_{i}^{02} E_{i+1}^{12}\right. \\
& \left.+\left(q+\frac{1}{q}\right) E_{i}^{01} E_{i+1}^{02}+\left(q+\frac{1}{q}\right) E_{i}^{02} E_{i+1}^{01}\right)
\end{aligned}
$$

The Hamiltonian $H_{0} / D^{\prime}=\sum_{i=1}^{L-1} e_{i}^{0}$ was introduced in [53], (see also Ref. [54] for the case $q=1$ ) as a $3^{L}$-dimensional representation of the Hecke algebra. We verified, guided by the physical processes of diffusion and annihilation, the the operators $e_{i}$ $(i=1, \ldots, L-1)$ also satisfy the same algebra. Morevover, we checked that both $e_{i}^{0}$ and $e_{i}$ satisfy the quotient (C.5) like the $U_{q} S U(1 / 1)$ chains, for the underlying quantum symmetry of (C.25) see Ref.55.

\section{Figure captions}

Figure 1: Phase diagram of the XXZ quantum chain with anisotropy $\Delta$ in a magnetic field $h . I$ is the massive (frozen) ferromagnetic phase, $I I$ the massless phase which 
is commensurate for $h=0$ and incommensurate for $h \neq 0$ and $I I I$ is the massive

(frozen) antiferromagntic phase. The line $\Delta=1-h$ corresponds to a PokrovskyTalapov transition.

Figure 2: Boltzmann weights of the two-dimensional vertex model associated to the Hamiltonian Eq. (B.1). The first column gives the weights, the second one the arrow diagram and the third one the relationship between the vertices and the elementary reaction-diffusion processes.

\section{References}

[1] A. Tsuchiya, T. Eguchi and M. Jimbo (Eds) "Infinite analysis", Part A and B, World-Scientific,Singapore, 1992.

[2] M.V. Smoluchowski, Z. Phys. Chem. 92 (1917), 129.

[3] A.A. Ovchinnikov and Y.B. Zeldovich, Chem. Phys. 28 (1978), 215.

[4] V. Kuzovkov and E. Kotomin, Rep. Prog. Phys. 51 (1988), 1479.

[5] L. Gálfi and Z. Rácz, Phys. Rev. A38 (1988), 3151.

[6] S. Cornell, B. Chopard and M. Droz, Phys. Rev. A44 (1991), 4826.

[7] B. Chopard and M. Droz, Europhys. Lett. 15 (1991), 459; Z. Jiang and C. Ebner, Phys. Rev. A42 (1990), 7483.

[8] M. Bramson and J. Lebowitz, J. Stat. Phys. 62 (1991), 297.

[9] D. Toussaint and F. Wilczek, J. Chem. Phys. 78 (1983), 2642.

[10] F. Leyvraz and S. Redner, Phys. Rev. A46(1992), 3132.

[11] K. Kang and S. Redner, Phys. Rev. A30 (1984), 2833.

[12] K. Kang and S. Redner, Phys. Rev. Lett. 52 (1984), 955.

[13] R. Schoonover, D. Ben-Avraham, S. Havlin, R. Kopelman and G. Weiss, Physica 171A (1991), 232.

[14] B. Chopard, M. Droz, T. Karapiperis and Z. Rácz, Phys. Rev. E, to appear (1993).

[15] K. Kawasaki, in Phase Transitions and Critical Phenomena, C. Domb and M.S. Green (Eds), Academic Press,New York 1972. 
[16] B.U. Felderhof, Rep. Math. Phys. 1 (1970), 219.

[17] S. Cornell and M. Droz, Phys. Rev. A , to appear (1993).

[18] M. Droz, J. Kamphorst Leal da Silva, A. Malaspinas and J. Yeomans, J. Phys. A19 (1986), 2671.

[19] R.J. Glauber, J. Math. Phys. 4 (1963), 294.

[20] L.P. Kadanoff and J. Swift, Phys. Rev. 165 (1968), 310 ;B.I. Halperin, P.C. Hohenberg and S.-K. Ma, Phys. Rev. B10 (1974), 139; P. Grassberger and M. Scheunert, Fortschritte der Physik 28, (1980), 547.

[21] V.R. Jones, Int. J. Mod. Phys. B4 (1990), 701.

[22] C.L. Schultz, Phys. Rev. Lett. 46 (1981), 629; Physica 122 (1983), 71; J.H.H. Perk and C.L. Schultz, in M. Jimbo and T. Miwa (Eds) Non-Linear Integrable Systems, Classical Theory and Quantum Theory, World Scientific, Singapore, 1981.

[23] P.P. Martin and V. Rittenberg, Int. J. Mod. Phys. A7, Suppl. 1B 1992), 707.

[24] D. Kandel, E. Domany and B. Nienhuis, J. Phys. A23 (1990), L755; L.-H. Guo and H. Spohn, Phys. Rev. Lett. 68 (1992), 725 ; G. Schütz, "Generealized Bethe Ansatz Solution of an One-Dimensional Asymmetric Exclusion Process on a Ring with Blockage",Preprint Weizmann 1992.

[25] F. C. Alcaraz and V. Rittenberg, to be published.

[26] V. Pasquier and H. Saleur, Nucl. Phys. B330 (1990), 523.

[27] V. L. Pokrovsky and A. L. Talapov, Sov. Phys. JETP, 51 (1980) 134.

[28] J.D. Johnson and B.M. McCoy, Phys. Rev. A6 (1972), 1613; Takanishi, Prog. Theor. Phys. 50 (1973), 1519; 51 (1974), 1348. M. Lüscher, Nucl. Phys. B117 (1976), 475; I. Affleck in E. Brézin and J. Zinn-Justin (Eds) Fields, Strings and Critical Phenomena, Les Houches XLIX, North Holland,Amsterdam 1990, p. 563.

[29] F.C. Alcaraz, M. Henkel and V. Rittenberg, to be published.

[30] D. ben-Avraham, M.A. Burschka and C.R. Doering, J. Stat. Phys. 60 (1990), 694.

[31] E. Siggia, Phys. Rev. B16 (1977), 2319.

[32] F. Family and J.G. Amar, J. Stat. Phys. 65 (1991), 1235. 
[33] V. Privman (Ed), Finite-size Scaling and Numerical Simulations of Statistical Systems, World Scientific, Singapore, 1990); P. Christe and M. Henkel, Introduction to Conformal Invariance and Its Applications to Critical Phenomena, Springer Lecture Notes in Physics, Vol. m16, Springer, Heidelberg, 1993.

[34] A.A. Lushnikov, Sov. Phys. JETP 64 (1986), 811; Phys. Lett. 120A (1987), 135.

[35] J.G. Amar and F. Family, Phys. Rev. A41 (1990), 3258.

[36] E.I. Ogievetsky, N.Yu. Reshetikin and P. Wiegmann, Nucl. Phys. B280 (1987), 45; N.Yu. Reshetikin, Teor. Mat. Fiz. 12 (1985), 347; S.V. Pokrovsky and A.M. Tsvelik, Sov. Phys. JETP 66 (1987), 1275.

[37] B. Sutherland, Phys. Rev. B12 (1975), 3795.

[38] N. Reshetikhin, Letters Math. Phys. 20 (1990), 331; P. Truini and V. S. Varadarajan, Letters Math. Phys. 26 (1992), 53.

[39] V. Privman, Phys. Rev. A46 (1992), 6140.

[40] C. Ahn and K. Shigemoto, "Onsager Algebra and Integrable Lattice Models", CLNS preprint 1990.

[41] D. Arnaudon and V. Rittenberg, "Quantum Chains with $U_{q}(s l(2))$ Symmetry and Unrestricted Representations", CERN preprint CERN-TH 1993.

[42] S. Ostlund, Phys. Rev. B24 (1981), 398; D.A. Huse, Phys. Rev. B24 (1981), 5180.

[43] G. Forgas, S.T. Chui and H.L. Frisch, Phys. Rev. B22 (1980), 415.

[44] E.J.S. Lage J. Phys. A18 (1985), 2289.

[45] G. von Gehlen and V. Rittenberg, Nucl. Phys. B 257 [FS24] (1985), 351.

[46] H. Saleur, in Proc. of Trieste Conference on Recent Developments in Conformal Field Theories, 1989.

[47] C.N. Yang, Phys. Rev. Lett. 19 (1967), 1312; R.J. Baxter, "Exactly Solved Models in Statistical Mechanics, Academic Press, New York, 1982.

[48] H.N.V. Temperley and E.H. Lieb, Proc. Roy. Soc. London, A322 (1971), 251.

[49] F.C. Alcaraz, M.N. Barber and M.T. Batchelor, Ann. of Phys. 182 (1988), 280.

[50] T. Deguchi, J. Phys. Soc. Jpn. 58 (1989), 3441; T. Deguchi and Y. Akutsu, J. Phys. A23 (1990), 1861; M.L. Ge, G.C. Liu and K. Hue, Mod. Phys. Lett. A6 (1991), 1173. 
[51] P.W. Anderson, Science 235 (1987), 1196.

[52] P.A. Bares and G. Blatter, Phys. Rev. Lett. 64 (1990), 2567; N. Kawakami and S.-K. Yang, Phys. Rev. Lett. 65 (1990), 2309.

[53] F.C. Alcaraz, R. Köberle and A. Lima-Santos, Int. J. Mod. Phys. A7 (1992), 7615 .

[54] C. Goméz and G. Sierra, Phys. Lett. 285B (1992), 126.

[55] F.C. Alcaraz, D. Arnaudon, V. Rittenberg and M. Scheunert, to be published. 